\title{
Forward modelling to determine the observational signatures of propagating slow waves for TRACE, SoHO/CDS, and Hinode/EIS
}

\author{
N. R. Owen, I. De Moortel, and A. W. Hood
}

\begin{abstract}
School of Mathematics and Statistics, University of St Andrews, North Haugh, St Andrews, Fife, KY16 9SS, Scotland, UK e-mail: nick@mcs.st-and.ac.uk
\end{abstract}

Received 19 August 2008 / Accepted 27 November 2008

\section{ABSTRACT}

\begin{abstract}
Context. The propagation and damping of slow MHD waves in the solar atmosphere are investigated by numerical simulations and forward modelling, with particular emphasis placed on waves with periodicities of the order of five minutes.

Aims. We extend a coronal model by adding an equilibrium temperature gradient allowing study of wave propagation from the transition region to the corona.

Methods. A 1D model is used that includes gravitational stratification and damping by thermal conduction, optically thin radiation, and compressive viscosity. Forward modelling of the simulation results, for both uniform and non-uniform equilibrium temperature profiles, is undertaken to establish the observational consequences of the physical processes involved for TRACE, SoHO/CDS, and Hinode/EIS

Results. The presence of thermal conduction causes a phase shift between the wave velocity, energy, and density. This shift may be observable by comparing Doppler velocity and intensity observations. Phase shifts are also seen between intensity observations by different instruments and between different spectral lines. This is an observational effect that arises due to the forward modelling process in which observations are synthesised, but it is not seen in the simulation results. Oscillations from the transition region are found to dominate the coronal emission for TRACE 171 A by nearly two orders of magnitude.
\end{abstract}

Key words. Sun: oscillations - Sun: corona - Sun: transition region

\section{Introduction}

Global coronal seismology was originally proposed by Uchida (1970). Roberts et al. (1984) suggested the use of local coronal seismology combining MHD wave theory and observations in order to determine properties of the solar corona. Wave parameters such as periods, amplitudes and propagation speeds are measured and compared to theoretical models to give estimates of, for example, the coronal magnetic field strength. Coronal seismology was limited in 1984 due to the spatial and temporal limits of observations, however, this changed with the launch of the satellites SoHO (Domingo et al. 1995) and TRACE (Handy et al. 1999). A large number of MHD waves have been observed in the solar atmosphere including both fast and slow mode oscillations. Overviews of observed waves can be found in Erdélyi et al. (2003); Aschwanden (2004); Wang (2004) and more recently in De Moortel $(2005,2006)$ and Nakariakov \& Verwichte (2005). An overview of MHD wave theory in the solar atmosphere is given by Roberts (2004).

Propagating intensity oscillations have been detected in the corona, firstly in polar plumes by Ofman et al. (1997) using SoHO/UVCS (Kohl et al. 1995) and by Deforest \& Gurman (1998) with SoHO/EIT (Delaboudinière et al. 1995). These intensity oscillations have been interpreted as propagating slow waves (Ofman et al. 1999, 2000). Such oscillations have also been observed along coronal loops with SoHO/EIT (Berghmans \& Clette 1999) as well as by TRACE (Nightingale et al. 1999; Schrijver et al. 1999; De Moortel et al. 2000). Two overviews with many detections of such oscillations are given in De Moortel et al. (2002c,a) and McEwan \& De Moortel (2006), where the former found intensity oscillations of $4.1 \pm 1.5 \%$ with average propagation speeds of $122 \pm 43 \mathrm{~km} \mathrm{~s}^{-1}$. They also found that such oscillations are quickly damped and are only detected $8.9 \pm 4.4 \mathrm{Mm}$ along the loop from one footpoint and are never detected between the loop apex and the other footpoint. De Moortel et al. (2002b) used wavelet analysis to determine the period of such oscillations. They found two distinct bands of periodicity of around three and five minutes. The three minute periodicities were predominately found above sunspots, whereas the five minute periodicities were found on the edge of active regions. They suggest the observed oscillations are caused by an underlying driver, with the waves propagating through the transition region into the corona. Using numerical simulations De Pontieu et al. (2005) found that the leakage of the global five minute p-modes is possible with inclined magnetic field lines. McEwan \& De Moortel (2006) suggested that the oscillations they observed are driven by leakage into the corona from the global five minute p-modes. Oscillations above sunspots have also been observed by O'Shea et al. (2002) and Brynildsen et al. (2003). Oscillations with periodicity of five minutes have been observed simultaneously with SoHO/EIT and TRACE $171 \AA$ (Robbrecht et al. 2001) as well as with TRACE $171 \AA$ and TRACE $195 \AA$ (King et al. 2003). Marsh et al. (2003) and Marsh \& Walsh (2006) detected oscillations with periodicity of five minutes using co-temporal and co-spatial observations from TRACE $171 \AA$ and SoHO/CDS. Oscillations were detected over a temperature range of $\log (T)=4.54$ to 6 , covering the chromosphere, transition region and corona.

Propagating acoustic waves are discussed by Klimchuk et al. (2004) in the context of coronal seismology. By using a combination of analytical theory, numerical modelling and data 
analysis they find that the main effects on intensity perturbations are due to pressure and temperature stratification and dissipation due to thermal conduction. They found that classical thermal conduction is sufficient to explain intensity perturbations in 1-2 MK loops and an enhanced value is not required. Nakariakov et al. (2000) presented a 1D theoretical model interpreting propagating intensity oscillations as slow magnetoacoustic waves. The model included viscosity, thermal conduction, gravity and non-linear effects and is further developed by Tsiklauri \& Nakariakov (2001). Numerical simulations of the damping of slow waves has been undertaken for the solar corona with an atmosphere of uniform equilibrium temperature (De Moortel \& Hood 2003, 2004; De Moortel et al. 2004). This work combined theoretically predicted damping rates with those observed by TRACE. They examined the effects of damping due to compressive viscosity, thermal conduction, optically thin radiation and mode coupling on slow waves. They also included gravitational stratification and field line divergence. Compressive viscosity, optically thin radiation and mode coupling were not capable of explaining the rapid damping observed, however, a combination of thermal conduction, gravitational stratification and field line divergence did. The uniform temperature equilibrium of one million Kelvin used in De Moortel \& Hood (2003, 2004); De Moortel et al. (2004), represents the solar corona. However, in order to study the propagation of these waves through the chromosphere and transition region and into the corona, an equilibrium temperature profile representing these regions of the solar atmosphere is required. This extension of the model used in De Moortel \& Hood (2003, 2004); De Moortel et al. (2004) allows the propagation and damping of slow waves to be examined through a larger part of the solar atmosphere rather than just in the corona.

Forward modelling is a useful technique where the plasma properties calculated in numerical simulations are used to determine the observable quantities of a region. As an example, the intensity of a region can be calculated, with some assumptions, from the plasma density and temperature. The forward modelling of 3D MHD simulations of the corona is discussed in Peter et al. (2006). Forward modelling has been used to study acoustic waves in the sub-photosphere (Shelyag et al. 2006) as well as standing slow modes in hot coronal loops (Taroyan et al. 2007). Taroyan \& Bradshaw (2008) compare forward modelling of a 1D loop model with observations from Hinode/EIS (Culhane et al. 2007) to investigate why standing slow modes have only been observed in hot coronal loops $(T>6 \mathrm{MK})$. Using forward modelling De Moortel \& Bradshaw (2008) found that determining the temperature and density perturbations from observations of coronal intensity oscillations was difficult and could easily lead to misinterpretation.

Using simulations we study waves with five minute periodicity, representing p-mode propagation from the solar surface. The effects of three damping mechanisms, namely, compressive viscosity, optically thin radiation and thermal conduction are included as in De Moortel \& Hood (2003, 2004), in addition to the effects of gravitational stratification. Slow waves are guided by magnetic field lines, hence their propagation is modelled in one dimension, assuming, for simplicity, a semi circular magnetic field. Observations of coronal oscillations have shown that perturbations in density are usually less then $10 \%$ of the background magnitude (De Moortel et al. 2002a). This process can, therefore, be modelled as linear. Hence, we are only concerned with the solution to the MHD equations for small amplitudes. The results of the simulations are forward modelled for both an imager (TRACE) and for spectrometers (SoHO/CDS and Hinode/EIS).
Section 2 describes the equations solved along with the numerical code used and the initial conditions of the model. The simulation and forward modelling results are discussed in Sects. 3 and 4, respectively and conclusions are presented in Sect. 5.

\section{Numerical model}

To simulate the propagation of slow waves in the solar atmosphere we solve the MHD equations of mass continuity, motion, energy and induction with an equation of state (Priest 1982). Including the terms for gravity, compressive viscosity, optically thin radiation and thermal conduction the equations are

$$
\begin{aligned}
\frac{\partial \rho}{\partial t}= & -\nabla \cdot(\rho \boldsymbol{v}) \\
\frac{\partial \boldsymbol{v}}{\partial t}= & -(\boldsymbol{v} \cdot \nabla) \boldsymbol{v}-\frac{1}{\rho} \nabla p+\frac{1}{\rho}(\boldsymbol{j} \times \boldsymbol{B})-\boldsymbol{g}+\frac{4}{3} \frac{v}{\rho} \nabla^{2} \boldsymbol{v}, \\
\frac{\partial \epsilon}{\partial t}= & -\boldsymbol{v} \cdot \nabla \epsilon-\frac{p}{\rho} \nabla \cdot \boldsymbol{v}+\frac{1}{\rho} \nabla\left(\kappa_{\|} \nabla_{\|} T\right)-\rho \tilde{\chi} T^{\alpha} \\
& +\frac{H_{0}}{\rho}+\frac{j^{2}}{\sigma \rho}+\frac{4}{3} \frac{v}{\rho}(\nabla \boldsymbol{v} \cdot \nabla \boldsymbol{v}), \\
\frac{\partial \boldsymbol{B}}{\partial t}= & -(\boldsymbol{v} \cdot \nabla) \boldsymbol{B}+\nabla \times(\boldsymbol{v} \times \boldsymbol{B})-\nabla \times(\eta \nabla \times \boldsymbol{B}), \\
\epsilon= & \frac{p}{(\gamma-1) \rho}
\end{aligned}
$$

where $\rho$ is the plasma density, $\boldsymbol{v}$ the plasma velocity, $t$ time, $p$ the plasma pressure, $\boldsymbol{j}$ the current density, $\boldsymbol{B}$ the magnetic field, $\boldsymbol{g}$ the gravitational acceleration, $v$ the coefficient of compressive viscosity, $\epsilon$ the specific internal energy, $T$ the plasma temperature, $\gamma$ the ratio of specific heat capacities of the plasma $\left(\gamma=5 / 3\right.$ in the corona, Priest 1982), $\kappa_{\|}$the coefficient of thermal conduction along magnetic field lines, $\sigma$ the electrical conductivity, $\eta$ the magnetic diffusivity and the coronal heating term, $H_{0}=\rho_{0}^{2} \tilde{\chi} T_{0}^{\alpha}+\frac{\partial}{\partial x}\left(\kappa_{\|} \frac{\partial T_{0}}{\partial x}\right)$, where $\tilde{\chi}$ and $\alpha$ are constants. It has been assumed that $\kappa_{\perp}<<\kappa_{\|}$.

We consider a constant vertical magnetic field along the $x$-axis which guides the wave, allowing a one-dimensional treatment. The equations are non-dimensionalised to the Alfvén speed (to be consistent with Arber et al. 2001) using a coronal density of $\rho_{00}=1.67 \times 10^{-12} \mathrm{~kg} \mathrm{~m}^{-3}$, a temperature of $T_{00}=10^{6} \mathrm{~K}$ and a wave period of $\tau=300 \mathrm{~s}$. By dropping bars from dimensionless quantities for clarity, Eqs. (1) to (4) become

$$
\begin{aligned}
\frac{\partial \rho}{\partial t}= & -\rho \frac{\partial v}{\partial x}-v \frac{\partial \rho}{\partial x} \\
\frac{\partial v}{\partial t}= & -v \frac{\partial v}{\partial x}-\frac{1}{\rho} \frac{\partial p}{\partial x}-G g+\frac{e}{\rho} \frac{\partial^{2} v}{\partial x^{2}} \\
\frac{\partial \epsilon}{\partial t}= & -v \frac{\partial \epsilon}{\partial x}-(\gamma-1) \epsilon \frac{\partial v}{\partial x}+\frac{d}{\rho} \frac{\partial}{\partial x}\left(\epsilon^{5 / 2} \frac{\partial \epsilon}{\partial x}\right) \\
& -r \rho \epsilon^{\alpha}+\frac{H_{*}}{\rho}+\frac{e}{\rho}\left(\frac{\partial v}{\partial x}\right)^{2}
\end{aligned}
$$

$\frac{\partial B}{\partial t}=0$

where

$$
\begin{aligned}
& e=\frac{4}{3} \frac{v_{00}}{v_{A 0}^{2} \tau \rho_{00}}, \\
& d=\left(\frac{(\gamma-1) \tilde{\mu}}{R}\right)^{7 / 2} \frac{\epsilon_{00}^{3 / 2} \kappa_{\| 00}}{\tau \rho_{00}},
\end{aligned}
$$




$$
\begin{aligned}
r & =\left(\frac{(\gamma-1) \tilde{\mu}}{R}\right)^{\alpha} \tilde{\chi} \tau \rho_{00} \epsilon_{00}^{(\alpha-1)}, \\
G & =\frac{g_{00} L}{\epsilon_{00}}
\end{aligned}
$$

and, to model a closed coronal loop, dimensionless gravity along $\boldsymbol{B}$ is taken as $g=\cos \left(\frac{\pi x}{L}\right)$. This represents a semi-circular coronal loop which is straightened and hence modelled in 1D. $R$ in Eqs. (11) and (12) is the ideal gas constant.

The values of $\rho_{00}, T_{00}, \tau$ and $\gamma$ from above as well as $\tilde{\mu}=0.6$ and a gravitational acceleration of $g_{00}=274 \mathrm{~m} \mathrm{~s}^{-2}$ give sound and Alfvén speeds of $c_{\mathrm{s}}=152 \mathrm{~km} \mathrm{~s}^{-1}$ and $v_{\mathrm{A}}=705 \mathrm{~km} \mathrm{~s}^{-1}$ respectively. In addition, this gives a magnetic field strength of $B=10.2 \mathrm{G}$ and a plasma beta of $\beta=0.056$, which are typical of the solar corona.

We use the Lare1d code, a finite volume, shock capturing code that solves the non-linear MHD equations, as described by Arber et al. (2001). Linear effects will be modelled using small wave amplitudes. The heating effects due to compressive viscosity are small in the linear regime as the term $\frac{e}{\rho}\left(\frac{\partial v}{\partial x}\right)^{2}$ in Eq. (8) is of order $\mathrm{O}\left(v^{2}\right)$ and can, therefore, be neglected.

The computational box contains a closed coronal loop of length 16 Alfvén wavelengths with both boundaries representing loop footpoints. The footpoints are not photospheric, but lie in the chromosphere/transition region due to temperature constraints on the model discussed later. The lower boundary is defined at $x=0$, whilst the upper is at $x=16$. A large diffuse coronal loop is modelled using only the first half of the box $(x \leq 8)$, with the simulation running until the wave front reaches the loop apex $(x=8)$. The wave velocity is driven at the lower boundary of the model as $v(x=0, t)=A \sin \omega t$, where, $\omega=2 \pi$. With the energy and density driven as $\epsilon(x=0, t)=\epsilon_{0}+B \sin \omega t$ and $\rho(x=0, t)=\rho_{0}+C \sin \omega t$, respectively. Quantities at the upper boundary are line-tied to their equilibrium values. This condition reflects waves from the top boundary, however, the simulations are stopped before these reflections modify the results.

In order to maintain an equilibrium, the coronal heating function, $H_{*}$, given in Eq. (8), must balance the background energy flow from the system due to thermal conduction and optically thin radiation. The condition for this to be satisfied is found by neglecting the time and velocity dependent terms of Eq. (8), which gives

$H_{*}=r \rho_{0}^{2} \epsilon_{0}^{\alpha}-d \frac{\partial}{\partial x}\left(\epsilon_{0}^{5 / 2} \frac{\partial \epsilon_{0}}{\partial x}\right)$.

For the case of a uniform temperature equilibrium, where $\frac{\partial \epsilon_{0}}{\partial x}=$ 0 , the conduction term in Eq. (14) is zero and hence, Eq. (14) reduces to

$H_{*}=r \rho_{0}^{2} \epsilon_{0}^{\alpha}$,

where the coronal heating term only has to balance losses due to optically thin radiation.

The uniform temperature equilibrium of $1 \mathrm{MK}$ used by De Moortel \& Hood (2003, 2004) represents the solar corona. However, to model wave propagation through the lower regions of the solar atmosphere into the corona, a non-uniform temperature profile is required. Figure 1 shows the profiles used, with temperature increasing from $\sim 300000 \mathrm{~K}$ through a transition region to a coronal value of $1.25 \mathrm{MK}$. The density decreases by two to three orders of magnitude due to gravitational stratification. To maintain a non-uniform temperature equilibrium, the

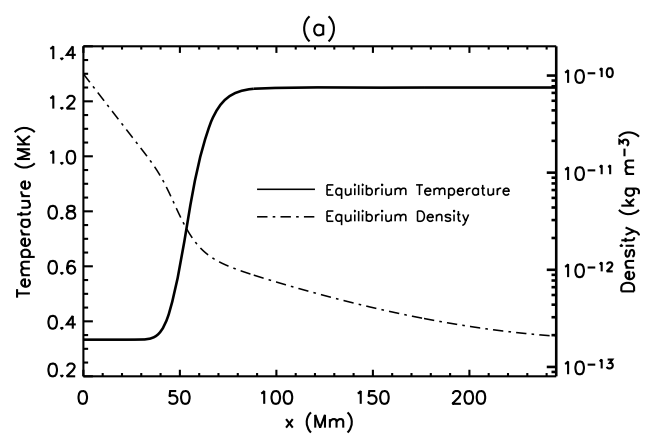

(b)

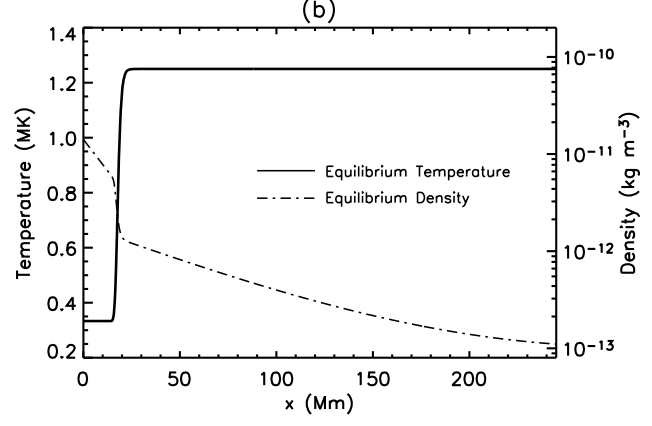

Fig. 1. Model atmospheres. a) Broad transition region and b) steep transition region.

initial conduction term, $d \frac{\partial}{\partial x}\left(\epsilon_{0}^{5 / 2} \frac{\partial \epsilon_{0}}{\partial x}\right)$, in Eq. (14), must be balanced as $\frac{\partial \epsilon_{0}}{\partial x} \neq 0$. This term is a function of $x$, but is independent of $t$. It is, therefore, calculated at time, $t=0$, using finite difference, and is added to the energy equation at every subsequent time step in order to maintain the initial temperature gradient.

Initially an equilibrium temperature profile with a broad transition region is used, as shown in Fig. 1a. The results from this are compared to a more realistically steep transition given by Fig. 1b. Due to the broad transition region in Fig. 1a the equilibrium density decreases by an order of magnitude more than in Fig. 1b, the effect of which is discussed in Sect. 3.2.

The two profiles shown in Fig. 1 are normalised to coronal conditions. That is, for both Figs. $1 \mathrm{a}$ and $\mathrm{b}$ at a temperature of $1 \mathrm{MK}$ the density is $1.67 \times 10^{-12} \mathrm{~kg} \mathrm{~m}^{-3}$. As a result, the density at $x=0$ of both profiles differs, as does the distance to the start of the temperature gradient $(x \approx 40 \mathrm{Mm}$ for (a) and $x \approx 10 \mathrm{Mm}$ for (b)).

For long wavelength oscillations with periodicity of a few minutes the transition region of the steep temperature gradient (Fig. 1b) approximates a discontinuity. By using the broad temperature profile, shown in Fig. 1a, the effects of adding a non-uniform temperature profile can be investigated without the additional complexity of a discontinuity. The effects of the discontinuity are then ascertained by comparing the results of the broad temperature gradient with those of the steep.

In order to model wave propagation from the solar surface a minimum temperature of around $6000 \mathrm{~K}$ is required. However, below $10^{4.5} \mathrm{~K}$, due to the high density the approximation of optically thin radiation becomes invalid i.e. the mean free path is shorter than typical length scales. Therefore, the model is restricted to propagation from the chromosphere into the corona. The minimum temperature currently used in the model is $300000 \mathrm{~K}$, which is limited due to numerical effects. These are, firstly, the steep temperature gradient which requires an increased spatial resolution and secondly, the high density contrast which reduces the numerical time step due to the CFL 
condition. The CFL condition in Lare1d is given by

$\delta t \leq \frac{\delta x}{c} \approx \delta x \sqrt{\frac{\rho}{\gamma p+B^{2}}}$,

which is proportional to $\sqrt{\rho}$.

One effect of underestimating the transition region temperature gradient is to reduce the amount of wave reflection. This is particularly true for the shallow gradient shown in Fig. 1a and to a lesser extent for Fig. 1b. A more realistically steep temperature gradient with increased wave reflection would produce waves with a lower initial amplitude in the corona than is found in Fig. 7. As a result, the detectable damping length may be reduced towards observed values (see Sects. 3.2 and 5).

\section{Simulation results}

\subsection{Uniform temperature equilibrium}

\subsubsection{Initial tests}

As an initial test, the code was run for a uniform equilibrium temperature of $1 \mathrm{MK}$, both with and without gravity. The analysis included both the separate and combined effects of compressive viscosity, optically thin radiation and thermal conduction. The results show damping rates and behaviour consistent with earlier work by De Moortel \& Hood (2003, 2004). In particular, it is confirmed that compressive viscosity and optically thin radiation cause some damping to waves in both uniform and gravitationally stratified media, but, for a uniform coronal medium (i.e., density $\approx 10^{-12} \mathrm{~kg} \mathrm{~m}^{-3}$ and temperature of $1 \mathrm{MK}$ ), the effect of these processes is very low compared to that of thermal conduction. An example of the uniform temperature equilibrium results are given in Fig. 2a, which shows a snapshot in time $(t=9.5)$ of the velocity of a propagating slow wave being damped in a uniform medium. The dot-dashed line in Fig. 2a results from the inclusion of all three damping mechanisms, whereas the solid line represents the velocity when only thermal conduction is included. The dotted line shows the corresponding undamped wave amplitude. The similarity between the two damping rates demonstrates the importance of thermal conduction compared to the damping due to viscosity and radiation. As a result, compressive viscosity and optically thin radiation have been neglected in the rest of this study. This was achieved by setting the coefficients of viscosity, $e$, in Eqs. (7) and (8) and that of radiation, $r$, in Eq. (8), to zero.

The inclusion of gravity results in an equilibrium density of the form $\rho_{0}=\hat{\rho_{0}} \exp \left(\frac{1}{H(x)}\right)$, where $H$ is the integrated gravitational scale height. This stratification causes the amplitude of the velocity to grow exponentially (see for example Roberts 1985; De Moortel \& Hood 2004) as given by

$v=\hat{v} \exp \frac{1}{2 H(x)}$

where $H$ is

$H(x)=\frac{\pi(\gamma-1) \epsilon_{0}}{L G \sin \left(\frac{\pi x}{L}\right)}$,

with loop length $L$ and equilibrium energy $\epsilon_{0}$. This exponential growth is shown in Fig. $2 b$ by the dot-dashed line, which matches the simulation results shown by a solid line.

The initial testing of the code for uniform equilibrium temperature and density has produced results for the three

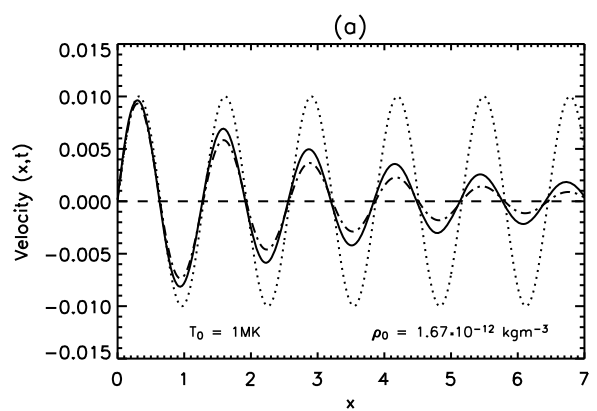

(b)

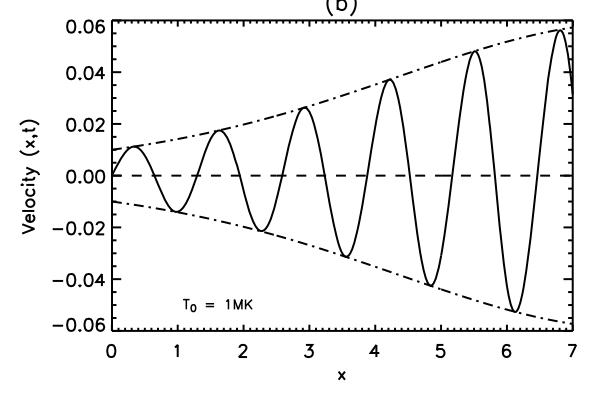

Fig. 2. Velocity of a sound wave. a) Undamped wave (dotted), with damping due to thermal conduction (solid) and damping due to all three mechanisms (dot-dashed). b) Undamped wave in a stratified medium with simulation results (solid) and predicted growth (dot-dashed) given by Eq. (17). Snapshot taken at time, $t=9.5$.

damping mechanisms that are consistent with De Moortel \& Hood $(2003,2004)$. In addition to this, gravity has been successfully included in the model with an exponential growth in wave amplitude found to agree with Roberts (1985). These tests confirm the accuracy of the numerical scheme and allow further analysis of the effects of thermal conduction as well as extending the model to a non-uniform temperature equilibrium.

\subsubsection{Thermal conduction}

Using a uniform equilibrium temperature of $1 \mathrm{MK}$, Fig. 3 shows the damping of a slow wave due to thermal conduction in an unstratified medium. The simulation results for velocity are represented in Fig. 3a by a solid line. The energy perturbation, $\epsilon_{1}$, is obtained from the code, at a particular snapshot in time, by subtracting the equilibrium value from the total energy. This can be written as $\epsilon_{1}=\epsilon-\epsilon_{0}$, where $\epsilon_{0}=\epsilon(t=0, x)$ and is represented in Fig. 3a by a dashed line. Observationally, oscillations in intensity, which are related to the density, are detected due to the relative amplitude of the perturbations compared to the background intensity. Consequently, the density, shown by a dot-dashed line in Fig. 3a, represents the perturbation relative to the background as given by $\frac{\rho-\rho_{0}}{\rho_{0}}=\frac{\rho_{1}}{\rho_{0}}$, where $\rho_{0}=\rho(t=0, x)$. The wave velocity has an initial maximum amplitude at $x=0$ of 0.01 (i.e. $v(x=0, t)=A \sin \omega t$ where $A=0.01)$, which was deliberately chosen to be small, to avoid non-linear effects as far as possible.

Previous work by De Moortel \& Hood $(2003,2004)$ looked mainly at the wave velocity, whereas we also want to investigate the perturbations in energy and density. Slow waves have been observed by measuring the intensity of emitted ultraviolet radiation from the corona, for example by TRACE (e.g. De Moortel et al. 2002a). This intensity is related to the density and temperature/energy of the medium. In contrast to this, Doppler velocity observations depend only on the velocity of the emitting region. The comparison between velocity, energy 
(a)

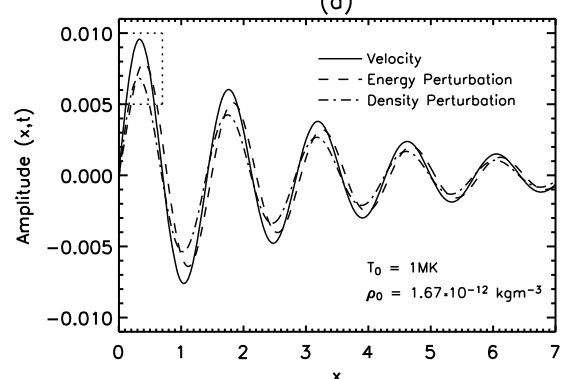

(b)

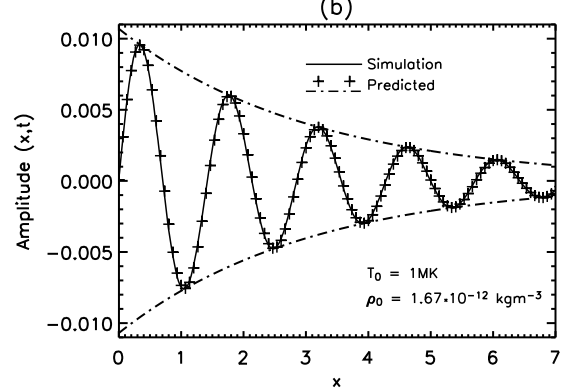

(c)

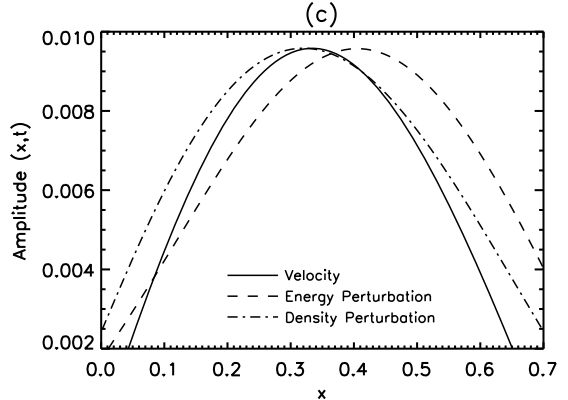

Fig. 3. Phase shift due to thermal conduction. a) Simulation results for velocity, density and energy. b) Simulation results for velocity (solid) with normal mode analysis prediction shown by crosses and dot-dashed lines. c) The dotted box in a) is enlarged with the energy and density amplitudes renormalised to illustrate the relative phase shift from the velocity more clearly. Snapshot taken at time, $t=9.5$.

and density may, therefore, have important observational consequences when analysing data of both intensity and Doppler velocity.

There are two noticeable effects due to thermal conduction shown in Fig. 3a. Firstly, all perturbed quantities $(v, \rho, \epsilon)$ are clearly damped as expected from the initial tests in Sect. 3.1.1. Secondly, there appears to be a phase shift between the velocity and the perturbations in energy and density. Without thermal conduction the three wave components travel in phase, but the inclusion of thermal conduction causes the peaks in density and energy to lag and lead the velocity, respectively. This phase shift may have consequences when comparing intensity and Doppler shift observations. Understanding the effects of thermal conduction for a uniform temperature equilibrium, in particular the phase shift seen in Figs. 3a and c, will aid our understanding of the non-uniform equilibrium effects, which are investigated in Sect. 3.2. In order to achieve this, a normal mode analysis is performed for thermal conduction in an unstratified medium which is combined with an analytical small parameter approximation to explain both the amplitude decay and the phase shifts noticed in Fig. 3.

In Fig. 3a, the density and energy perturbations are out of phase relative to the velocity by some phase shift $x_{\rho}$ and $x_{\epsilon}$, respectively. To isolate the effect of thermal conduction, we neglect gravity, viscosity and radiation $(G, e, r=0)$ and linearise Eqs. (6) to (8) to give

$$
\begin{aligned}
\frac{\partial \rho_{1}}{\partial t} & =-\rho_{0} \frac{\partial v}{\partial x}, \\
\frac{\partial v}{\partial t} & =-\frac{1}{\rho_{0}} \frac{\partial p_{1}}{\partial x}, \\
\frac{\partial \epsilon_{1}}{\partial t} & =-(\gamma-1) \epsilon_{0} \frac{\partial v}{\partial x}+\frac{d}{\rho_{0}} \frac{\partial}{\partial x}\left(\epsilon_{0}^{5 / 2} \frac{\partial \epsilon_{1}}{\partial x}\right) .
\end{aligned}
$$

Assuming $v, \rho_{1}$ and $\epsilon_{1}$ have the form $\mathrm{e}^{\mathrm{i}(k x-\omega t)}$ and using Eq. (5), the above equations become

$$
\begin{aligned}
\omega \rho_{1}-k \rho_{0} v & =0, \\
\omega v-(\gamma-1) k \frac{\epsilon_{0}}{\rho_{0}} \rho_{1}-(\gamma-1) k \epsilon_{1} & =0, \\
\left(\omega+\mathrm{i} d k^{2} \frac{\epsilon_{0}^{5 / 2}}{\rho_{0}}\right) \epsilon_{1}-(\gamma-1) \epsilon_{0} k v & =0,
\end{aligned}
$$

which can be expressed in matrix form as

$$
\left[\begin{array}{ccc}
\omega & -k \rho_{0} & 0 \\
-(\gamma-1) k \frac{\epsilon_{0}}{\rho_{0}} & \omega & -(\gamma-1) k \\
0 & -(\gamma-1) \epsilon_{0} k & \omega+\mathrm{i} d k^{2} \frac{\epsilon_{0}^{5 / 2}}{\rho_{0}}
\end{array}\right]\left[\begin{array}{c}
\rho_{1} \\
v \\
\epsilon_{1}
\end{array}\right]=0
$$

For a unique solution, the determinant of the matrix above has to equal zero, which gives

$k= \pm \sqrt{-\frac{C}{2 B} \pm \sqrt{\left(\frac{C}{2 B}\right)^{2}-\frac{D}{B}}}=k_{r}+\mathrm{i} k_{i}$,

where

$$
\begin{aligned}
& B=\mathrm{i}(\gamma-1) d \frac{\epsilon_{0}^{7 / 2}}{\rho_{0}}, \\
& C=\gamma(\gamma-1) \epsilon_{0} \omega-\mathrm{i} \omega^{2} d \frac{\epsilon_{0}^{5 / 2}}{\rho_{0}}, \\
& D=-\omega^{3} .
\end{aligned}
$$

As $k$ is complex we can write, $k=r \mathrm{e}^{\mathrm{i} \phi}$ where

$r=\sqrt{k_{r}^{2}+k_{i}^{2}}, \quad \phi=\tan ^{-1}\left(\frac{k_{i}}{k_{r}}\right)$.

Hence, using Eq. (22), the perturbed density can be written as

$\rho_{1}=A \frac{r \rho_{0}}{\omega} \mathrm{e}^{\mathrm{i} k_{r}\left(x+x_{\rho}\right)} \mathrm{e}^{-k_{i} x}$,

where $A$ is the initial amplitude of velocity $\left(v=A \mathrm{e}^{\left(\mathrm{i} k_{r} x-k_{i} x\right)}\right)$ and $x_{\rho}$ is the phase shift of $\rho_{1}$ with respect to $v$, given by

$x_{\rho}=\frac{\phi}{k_{r}}$.

Similarly, the energy perturbation is found by rearranging Eq. (23) to give

$\epsilon_{1}=\frac{\omega v}{(\gamma-1) k}-\frac{\epsilon_{0}}{\rho_{0}} \rho_{1}$.

By substituting for $k, \rho_{1}$ and $v$ this becomes

$\epsilon_{1}=A \mathrm{e}^{\mathrm{i} k_{r} x} \mathrm{e}^{-k_{i} x}\left(\alpha \mathrm{e}^{-\mathrm{i} \phi}-\beta \mathrm{e}^{\mathrm{i} \phi}\right)=A R \mathrm{e}^{\mathrm{i} k_{r}\left(x+x_{\epsilon}\right)} \mathrm{e}^{-k_{i} x}$, 
where

$R=\sqrt{(\alpha-\beta)^{2} \cos ^{2} \phi+(\alpha+\beta)^{2} \sin ^{2} \phi}$,

$\Phi=\tan ^{-1}\left(-\frac{(\alpha+\beta) \sin \phi}{(\alpha-\beta) \cos \phi}\right)$,

$\alpha=\frac{\omega}{(\gamma-1) r}, \beta=\frac{\epsilon_{0} r}{\omega}$,

with a phase shift of

$x_{\epsilon}=\frac{\Phi}{k_{r}}$.

Figure $3 \mathrm{~b}$ shows the velocity of a wave in a uniform medium damped by thermal conduction. The solid line represents the simulation results with crosses giving the predicted velocity from the normal mode analysis discussed above. The dot-dashed line shows the exponential damping given by exp $\left(-k_{i} x\right)$, derived from the above analysis. In addition to velocity, the results of the normal mode analysis predict the perturbations in energy and density, including the relative phase difference between them. For clarity only the velocity is plotted in Fig. $3 \mathrm{~b}$.

The phase shifts, $x_{\rho}$ and $x_{\epsilon}$, can be estimated by using a small parameter approximation, where the thermal conduction constant, $d$, is assumed small and hence terms of order $\mathrm{O}\left(d^{2}\right)$ can be neglected. Setting $k=k_{r}+\mathrm{i} k_{i}=\frac{\omega}{c_{\varsigma}}+\mathrm{i} d \Omega$ and substituting $k$ into the determinant of matrix (A), solving for $\Omega$ gives

$\Omega=\frac{\omega^{2}}{2 \gamma^{5 / 2}(\gamma-1)^{1 / 2}} \frac{\epsilon_{0}}{\rho_{0}}$.

Using Eqs. (29) and (31), the phase shift for density, $x_{\rho}$, is given by

$x_{\rho}=\frac{1}{k_{r}} \tan ^{-1}\left(\frac{k_{i}}{k_{r}}\right) \approx \frac{c_{\mathrm{s}}}{\omega} \tan ^{-1}\left(\frac{d \Omega c_{\mathrm{s}}}{\omega}\right) \approx \frac{d \Omega c_{\mathrm{s}}^{2}}{\omega^{2}}$,

which, using Eq. (38), becomes

$x_{\rho} \approx \frac{d \sqrt{(\gamma-1)}}{2 \gamma^{3 / 2}} \frac{\epsilon_{0}^{2}}{\rho_{0}}$.

The energy perturbation is given by substituting $k, \rho_{1}, v$ and $\Omega$ in to Eq. (32) to give,

$\epsilon_{1}=\sqrt{\frac{(\gamma-1) \epsilon_{0}}{\gamma}}-i \frac{(\gamma+1) d \omega}{2 \gamma^{5 / 2}(\gamma-1)^{1 / 2}} \frac{\epsilon_{0}^{2}}{\rho_{0}}$.

The relative phase shift from velocity is then given by

$x_{\epsilon}=\frac{1}{k_{r}} \tan ^{-1}\left(\frac{\operatorname{Im}\left\{\epsilon_{1}\right\}}{\operatorname{Re}\left\{\epsilon_{1}\right\}}\right) \approx-\frac{(\gamma+1) d}{2 \gamma^{5 / 2}(\gamma-1)^{1 / 2}} \frac{\epsilon_{0}^{2}}{\rho_{0}}$.

From Eqs. (40) and (42) we can see that both $x_{\rho}$ and $x_{\epsilon}$ have an $\frac{\epsilon_{0}^{2}}{\rho_{0}}$ dependence. This dependence of $x_{\epsilon}$ and $x_{\rho}$ on $\epsilon_{0}^{2}$ at constant $\rho_{0}$ can be seen in Fig. $4 \mathrm{a}$ and b. Figure $4 \mathrm{c}$ and $\mathrm{d}$ show the $\frac{1}{\rho_{0}}$ dependence of $x_{\epsilon}$ and $x_{\rho}$, respectively, for constant $\epsilon_{0}$. The values of $x_{\epsilon}$ and $x_{\rho}$ are measured from the simulations and shown in Fig. 4 by symbols. These values are measured to the nearest grid cell, hence there is an associated error on $x_{\epsilon}$ and $x_{\rho}$ of the order of the grid spacing. This is represented in Fig. 4 by vertical error bars. The analytical approximations are represented in Fig. 4 by dotted lines and agree with $x_{\epsilon}$ and $x_{\rho}$ from the normal mode analysis (dashed). Both calculated results agree with
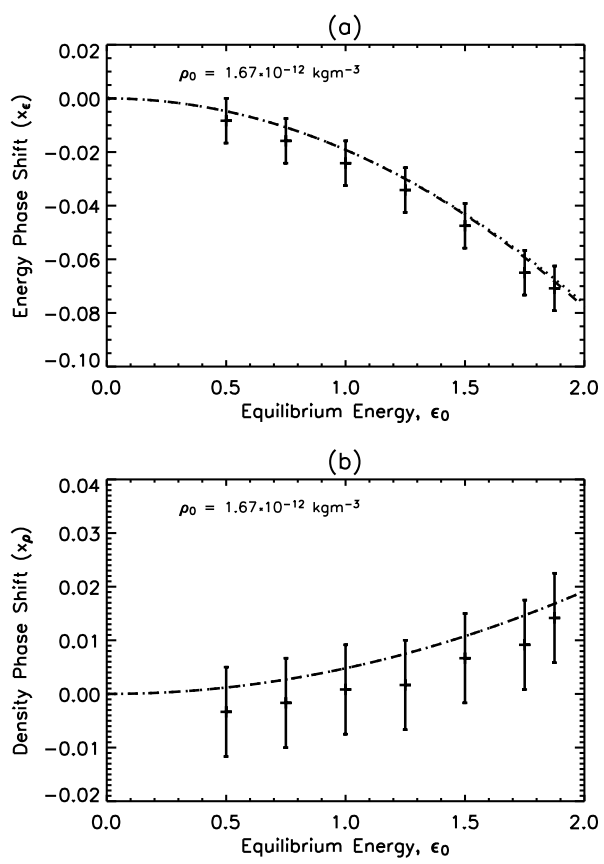

(c)

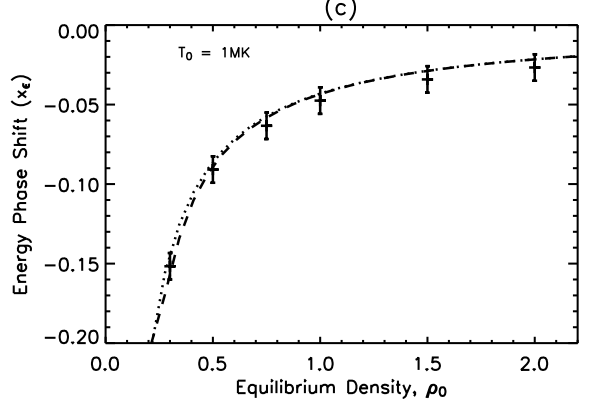

(d)

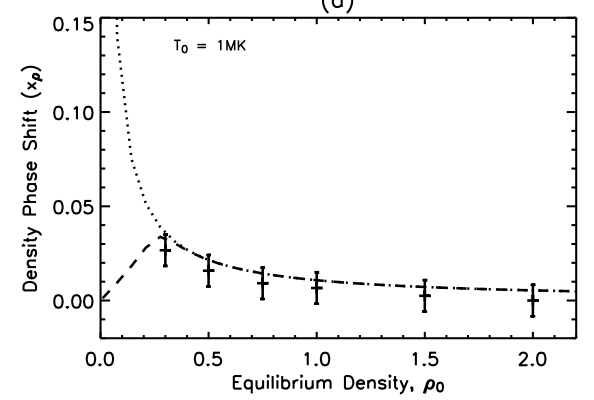

Fig. 4. Phase shift due to thermal conduction. Phase shift from normal mode analysis (dashed), with analytical approximation (dotted) and the measured phase shift with error bars, a) and b) give $x_{\epsilon}$ and $x_{\rho}$ respectively as a function of the equilibrium energy, c) and d) give $x_{\epsilon}$ and $x_{\rho}$ respectively as a function of the equilibrium density.

the measured results within the range of the error bars, except for the low density values of Fig. 4d. At this point the analytical approximation diverges from the normal mode analysis. This is a consequence of the assumption of small $d$, which becomes invalid when density is very low. From Eqs. (40) and (42), $x_{\rho}$ is positive, whereas, $x_{\epsilon}$ is negative, as shown by Fig. 4. This implies that the density lags the velocity, whereas the energy leads. This is in agreement with the simulation results, shown in Fig. 3a. This phase shift between $v$ and the perturbations in $\rho$ and $\epsilon$ occurs immediately after they are driven at the lower boundary. 
The propagation or sound speed is given by

$c_{\mathrm{s}}=\sqrt{\gamma(\gamma-1) \epsilon_{0}}$,

which is, therefore, constant in a region of uniform equilibrium temperature/energy. As a result, $v, \epsilon_{1}$ and $\rho_{1}$ propagate at the same speed and hence, the initial phase shift is maintained.

The excellent agreement between the simulation results and the predictions from both the normal mode analysis and the analytical approximation gives confidence in the treatment of thermal conduction. This allows the model to be extended to analyse the effects of thermal conduction with both gravity and a nonuniform equilibrium temperature.

\subsubsection{Thermal conduction and the effects of density}

Section 3.1.2 analysed the effects of thermal conduction in a medium of uniform density. Here we investigate firstly different uniform densities and secondly, a non-uniform density profile caused by the inclusion of gravity. The thermal conduction term in Eq. (8) is proportional to the inverse of the density and consequently, we can expect the behaviour of thermal conduction to be modified by the equilibrium density. Figure 5 shows slow waves in uniform media including the effects of thermal conduction for two uniform densities. Figure 5 a has the same density as Fig. 3a of $\rho_{0}=1.67 \times 10^{-12} \mathrm{~kg} \mathrm{~m}^{-3}$, with a lower density of $\rho_{0}=5.01 \times 10^{-14} \mathrm{~kg} \mathrm{~m}^{-3}$ in Fig. $5 \mathrm{~b}$.

At low densities, the thermal conduction term in Eq. (8) becomes large and hence, the effects of thermal conduction become very strong. Thermal conduction acts to smooth temperature gradients, which when density is low it does very efficiently. This can be seen by the energy perturbation (dot-dashed) in Fig. 5b, which is damped far more than that seen in Fig. 5a. As a result, if the density is sufficiently low, there is no longer an energy perturbation and the medium becomes isothermal. However, care must be taken when considering the effects of thermal conduction at such low densities. Luciani et al. (1983) consider non-local effects and question the validity of classical thermal conduction in this regime.

The wavelength of the propagating wave is given by $\lambda=\frac{P c_{\mathrm{s}}}{2 \pi}$, where the wave period, $P=2 \pi$. As a result, $\lambda=c_{\mathrm{s}}$. In an isothermal medium the wave propagates at the isothermal sound speed, $c_{s i}$, which is a factor of $\sqrt{\gamma}$ slower than the adiabatic sound speed, $c_{\mathrm{sa}}$ (De Moortel \& Hood 2003). The vertical dashed lines in Fig. 5a have a spatial separation of one wavelength based on the adiabatic sound speed, whereas, in Fig. 5b the isothermal sound speed is used. The peaks in velocity match the vertical lines in both figures, which shows that the wave in Fig. 5a is travelling at the adiabatic sound speed, whilst Fig. 5b travels almost at the isothermal sound speed. There is still a small energy perturbation in Fig. 5b, hence, the medium is not completely isothermal so the wave does not propagate at exactly the isothermal sound speed.

From the normal mode analysis, the damping due to thermal conduction is given by $\exp \left(-k_{i} x\right)$, as shown in Fig. $3 \mathrm{~b}$. The damping length, $\frac{1}{k_{i}}$, is, therefore, the distance over which the wave amplitude is damped by a factor of $\frac{1}{e}$. Using the normal mode analysis, this damping length can be calculated as a function of density and is shown by the solid line in Fig. 6a. The vertical dashed line represents the density that corresponds to the minimum damping length, that is the maximum rate of damping.

Thermal conduction damps perturbations by smoothing temperature gradients. As the temperature increases (or the density decreases) thermal conduction becomes more efficient at
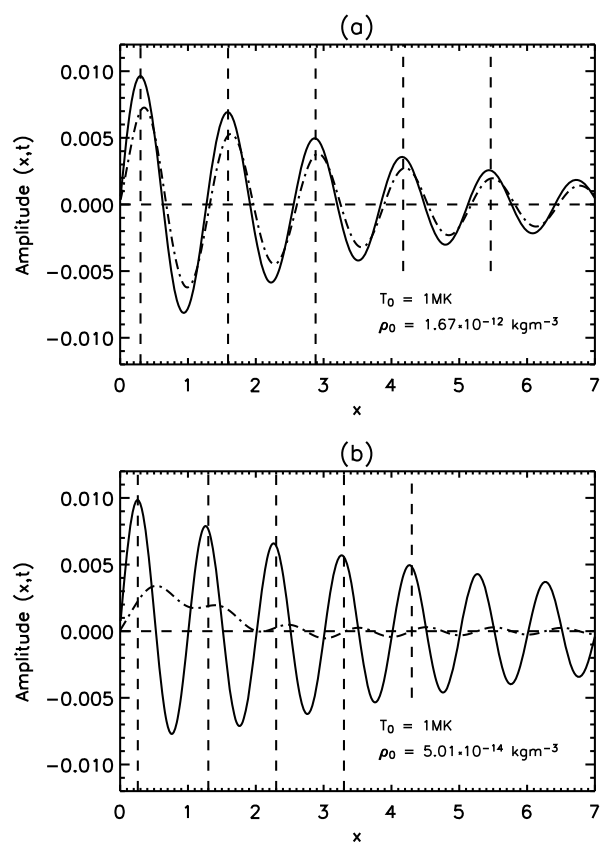

Fig. 5. Effects of density on thermal conduction. Velocity (solid) with the energy perturbation (dot-dashed). The dashed vertical lines show one wavelength when the wave is propagating at a) the adiabatic sound speed and b) the isothermal sound speed.

smoothing these gradients. As a result the temperature gradients are very quickly damped and the medium tends towards being isothermal, as shown for low density in Fig. 5b. The lack of temperature gradients in the isothermal medium prevents damping due to thermal conduction, hence, there is a maximum damping rate, or minimum damping length. In Fig. 6a the minimum damping length is at a density of $\rho_{0} \approx 0.25$. Above or below this density, thermal conduction is less efficient at damping the wave velocity. The minimum damping length due to thermal conduction is also discussed in De Moortel \& Hood (2003).

The inclusion of gravity produces a continually changing equilibrium density profile, which results in the effects of thermal conduction changing along the loop. For the case of a uniform equilibrium temperature of $1 \mathrm{MK}$, the equilibrium density at $x=0$ is normalised to unity (i.e $\rho_{0}=1.67 \times 10^{-12} \mathrm{~kg} \mathrm{~m}^{-3}$ ) and by including gravity it decreases exponentially with a value of $\rho_{0} \approx 0.03$ at $x=7$. There is a clear minimum damping length, shown in Fig. $6 \mathrm{a}$, at $\rho_{0} \approx 0.25$, which corresponds to $x \approx 2$ as seen in Fig. 6c. As a result, either side of this density the damping due to thermal conduction will be reduced. This result can be seen from the solid line in Fig. 6b which shows the velocity damped by thermal conduction in a stratified medium. The velocity is moderately damped initially before the damping rate is increased between $x=2$ and $x=3$ after which it remains relatively undamped. This is shown more clearly in Fig. 6c, where the damping length is plotted as a function of $x$. The fast reduction in damping is explained by the steep increase in damping length at densities lower than $\rho_{0} \approx 0.1$, corresponding to $x \geq 3.5$. The dot-dashed line in Fig. $6 \mathrm{~b}$ shows the damping due to thermal conduction for a uniform equilibrium density of 0.9 . The case with gravity (solid) damps quicker than with $\rho_{0}=0.9$ (dot-dashed) at $x \approx 2.5$ when thermal conduction is most efficient. However, the uniform density case damps quicker at both greater and lower $x$ as a result of the reduced damping of the case with gravity. 
(a)

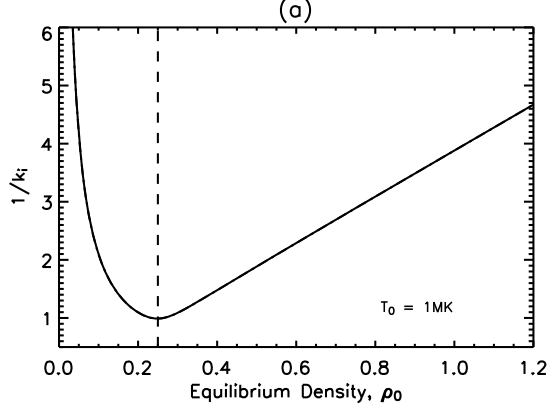

(b)

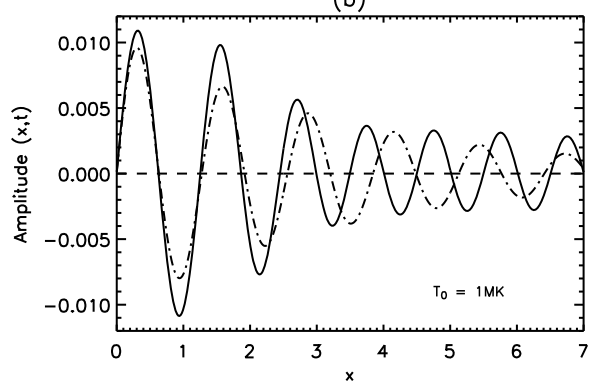

(c)

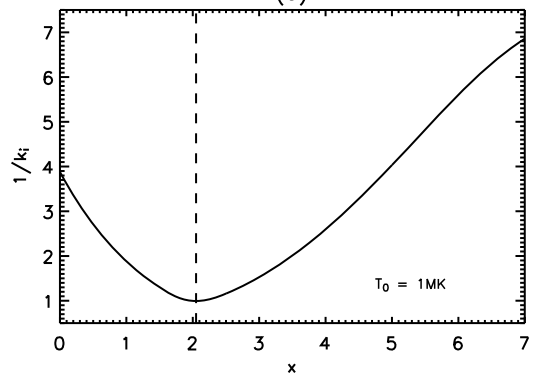

Fig. 6. Damping due to thermal conduction in a gravitationally stratified medium with a uniform temperature equilibrium. a) Damping length due to thermal conduction as a function of equilibrium density (solid), with the density of the minimum damping length (dashed). b) Velocity of a sound wave damped by thermal conduction with gravity (solid) and with a uniform equilibrium density of 0.9 (dot-dashed). Snapshot taken at time, $t=9.5$. c) same as a) but plotted as a function of $x$ rather than density.

The peaks in velocity for the case with gravity are seen in Fig. $6 \mathrm{~b}$ to progressively lag the uniform density case. This demonstrates how the increase in thermal conduction causes the wave to propagate at the isothermal sound speed and no longer at the faster adiabatic sound speed.

\subsection{Non-uniform equilibrium temperature}

In the previous section we investigated the effects of thermal conduction for uniform equilibrium temperature in both stratified and unstratified atmospheres. We found that thermal conduction can have a major effect on propagating slow waves. It causes rapid damping as well as a phase shift between the velocity and the perturbations in energy and density, as shown in Fig. 3. We now extend our study to a non-uniform equilibrium temperature (see Fig. 1) to investigate the effect of thermal conduction on wave propagation in a medium with changing background temperature and density.

Figure $7 \mathrm{a}$ and $\mathrm{b}$ show the simulation results for waves propagating in a gravitationally stratified medium with thermal conduction present with the equilibrium temperature and density

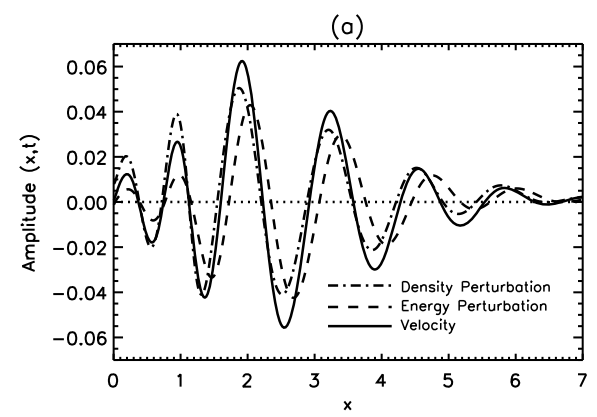

(b)

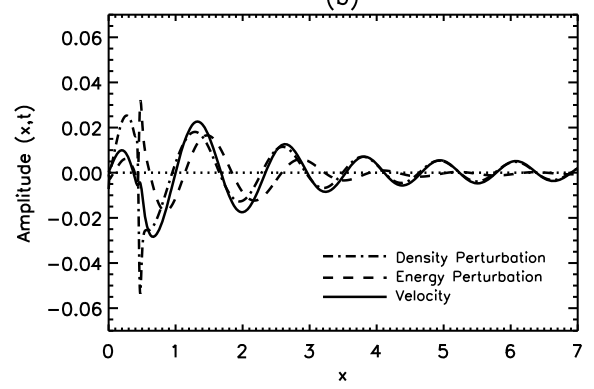

Fig. 7. Sound waves in a gravitationally stratified medium with a nonuniform temperature equilibrium. a) and b) with equilibrium temperature and density profiles as shown in Fig. 1a and b respectively.

profiles as shown in Fig. 1a and b, respectively. The difference between the two simulations shown in Fig. 7a and $b$ is the gradient of the temperature profile and the position of this gradient. Figure 7a has a shallow temperature gradient at $x \approx$ 1.7, whereas, Fig. $7 \mathrm{~b}$ has a more realistically steep gradient at $x \approx 0.5$.

For long wavelength oscillations with periodicity of a few minutes the transition region of the steep temperature gradient (Fig. 1b) approximates a discontinuity. By using the broad temperature profile, shown in Fig. 1a, the effects of adding a non-uniform temperature profile can be investigated without the additional complexity of a discontinuity. The effects of the discontinuity are then ascertained by comparing the results of the broad temperature gradient with those of the steep gradient.

In Fig. $6 \mathrm{~b}$ the damping due to thermal conduction dominates the increase in amplitude due to gravity and hence the wave amplitude decreases. The damping due to thermal conduction depends on the equilibrium density and temperature, as discussed in Sect. 3.1.3. Below the transition region ( $x \leq 1.7$ for Fig. $7 \mathrm{a}$ and $x \leq 0.5$ for Fig. $7 b$ ) the density is higher and the temperature lower than that of Fig. 6b. As a result the damping due to thermal conduction is less efficient. This, combined with the effect of gravity, causes the wave amplitude in both Fig. 7a and b to grow for small $x$. This growth continues until the transition region when the increase in temperature and reduction in density increases the efficiency of thermal conduction. The wave is then damped above the transition region. For pressure to remain constant the increase in temperature in the transition region is balanced by a decrease in density, which is in addition to that from gravity.

The main difference between the oscillations in Fig. 7a and b is the magnitude of the wave amplitude. Both are driven at $x=0$ with the same amplitude of 0.01 , yet the maximum amplitude in Fig. 7a is nearly three times that of Fig. 7b. This difference occurs due to the position of the transition region. With a transition region at $x \approx 1.7$, the wave in Fig. 7a experiences a stonger decrease in density due to gravity than Fig. $7 \mathrm{~b}$ where the transition 
region is at $x \approx 0.5$. Consequently, the wave amplitude in Fig. 7a grows larger before reaching the transition region where rapid damping due to thermal conduction begins. Another reason for the difference in amplitudes is due to reflection. The steep transition region of Fig. $7 \mathrm{~b}$ reflects more of the wave amplitude than that of Fig. 7a. As a result of these two effects, the amplitude of the wave in the coronal region $(x>1)$ of Fig. $7 \mathrm{~b}$ is much less than Fig. 7a and hence, would be observable for a shorter length.

A major contrast between Fig. 7a and $b$ are the spikes seen in the energy and density perturbations of Fig. 7b but not Fig. 7a. The perturbed quantities, $\epsilon_{1}$ and $\rho_{1}$ depend on the derivatives of the equilibrium quantities, $\epsilon_{0}$ and $\rho_{0}$. As a result, the large gradient in the equilibrium quantities in Fig. $1 \mathrm{~b}$ produces spikes in the density and energy perturbations which do not occur for the smaller derivatives of Fig. 1a.

The inclusion of thermal conduction, as discussed in Sect. 3.1.2, introduces a phase shift near the lower boundary $(x \approx 0)$. Using either temperature profile shown in Fig. 1 gives a propagation speed (Eq. (43)) that increases with $x$ and hence, at any given instance in time, features at larger $x$ will travel faster. Consequently, the initial phase shift will increase as, say a maximum of the energy perturbation will lead the corresponding velocity maximum and hence has a higher propagation speed (see Fig. 7). The increased phase shift between the perturbed quantities provides a larger possibility of observation by comparing the relative position of perturbations in intensity with those from Doppler shifts. Assessing the feasibility of such is beyond the scope of the present work.

The results of the simulations, as shown for example in Fig. 7, describe the velocity, energy and density of the system. These quantities allow some understanding of the physics behind the model, however, a more comprehensive understanding can be gained by comparison with observations. Velocities can be measured from Doppler shifts, however, the energy and density can not be directly observed. Consequently, a method that allows a comparison between the simulated and observed quantities is required.

\section{Forward modelling}

Forward modelling provides a valuable link between numerical simulations and observations. This technique determines the observable quantities of theoretical models and, therefore, allows a better understanding of the observational consequences and signatures of the physical processes shown by the simulation results. In addition it allows a more direct comparison between numerical results and observational data. Forward modelling can be performed for any observable quantity, for example Doppler velocity or intensity. This work, however, only considers intensity observations.

\subsection{Forward modelling code}

Forward modelling produces a synthesised emission that would be observed if a particular instrument (for example TRACE) was observing a region with conditions given by the model, in this case a coronal loop. Forward modelling for intensity observations requires the electron number density and the temperature in order to determine the synthesised emission along the loop $\left(\mathrm{DN} \mathrm{s}^{-1}\right.$ pixel $\left.^{-1}\right)$. In addition to the electron number density and the temperature, the ability of forward modelling in calculating synthesised emission is heavily dependent on atomic data and chemical abundances. The appropriate instrument response function is required, in addition to the emissivities of each element and ionisation state within the wavelength range of the instrument response function. The emissivities are obtained from the atomic data collaboration CHIANTI (Dere et al. 1997; Young et al. 2003). This information is stored for a range of temperatures and electron number densities and is linearly interpolated for the exact density and temperature from the simulation. Solar abundances are taken from Feldman et al. (1992), (equilibrium) ionisation balances from Mazzotta et al. (1998) and a line of sight depth of $5 \mathrm{Mm}$ is assumed. The emissivity data is converted into units of $\mathrm{DN} \mathrm{s}^{-1}$ pixel $^{-1} \mathrm{~cm}^{6}$, using,

$$
\mathrm{IU}\left(Y_{i}, \lambda, x\right)=\frac{0.83 \times \mathrm{AB} \times \mathrm{LOS} \times \mathrm{IRF} \times \lambda \times \mathrm{Em} \times Y_{i}}{4 \pi h c n_{\mathrm{e}}},
$$

where, IU is related to the emissivity, Em, $i$ is the population fraction of the $i$ th ionisation state of element $Y, \mathrm{AB}$ the atomic abundance normalised to that of hydrogen, LOS the line of sight depth and IRF the value of the instrument response function at the wavelength, $\lambda . h$ is Plank's constant, $n_{\mathrm{e}}$ the electron number density, $T_{\mathrm{e}}$ the (electron) temperature and $c$ the speed of light, where 0.83 is the proton/electron ratio (Bradshaw \& Mason 2003). IU is then summed over all ionisation states and wavelengths to give $\operatorname{IU}(x)$ as a function of loop position, which is multiplied by the number density squared to give intensity, $I(x)$ in units of $\mathrm{DN} \mathrm{s}^{-1}$ pixel $^{-1}$.

As discussed in Sect. 2, small amplitude perturbations were required to ensure the simulations modelled linear behaviour. Consequently, the energy and density from the simulations have perturbations of around $1 \%$ of the equilibrium value. However, observed amplitudes of propagating slow waves are around 5\% (De Moortel et al. 2002c), therefore, our perturbation amplitudes are multiplied by a factor of 5 . This results in oscillations of around 5\% in energy and density with the same equilibrium value. This multiplication assumes that the physical processes involved scale exactly linearly. However, other effects may become important when larger amplitudes are considered.

De Moortel \& Bradshaw (2008) used forward modelling of given temperature and density perturbations to investigate the resulting intensity perturbations for TRACE and Hinode/EIS. They do not model particular damping mechanisms, but used simple analytical expressions for the temperature and density with oscillations that decay in time or space.

Forward modelling can be applied to both imagers and spectrometers. Imagers, such as TRACE, have broad instrument response functions (relative to spectrometers) covering a wide temperature range. In contrast, spectrometers have narrow instrument response functions and, therefore, observe spectral lines emitted at more specific temperatures. The use of different spectral emission lines, which are formed at a range of temperatures, allows observations of different regions of the solar atmosphere. This allows analysis of, in this case, slow waves as they propagate through the solar atmosphere. The simulation results are modelled for the imager TRACE in the $171 \AA$ passband in addition to the spectrometers SoHO/CDS Mg X (624 $\AA$ ) and Hinode/EIS Fe XII (195 ̊) and EIS Mg VI (269 ̊). These lines have been chosen to cover a temperature range from the transition region (Mg VI, $T \approx 0.4 \mathrm{MK}$ ) to the corona (Fe XII, $T \approx 1.25 \mathrm{MK}$ ), which corresponds nicely with the temperature profile shown in Fig. 1.

Intensity observations and, therefore, the synthesised emission produced by forward modelling depends on contributions from many different factors. To understand the observational consequences of propagating slow waves in a gravitationally 
(a)

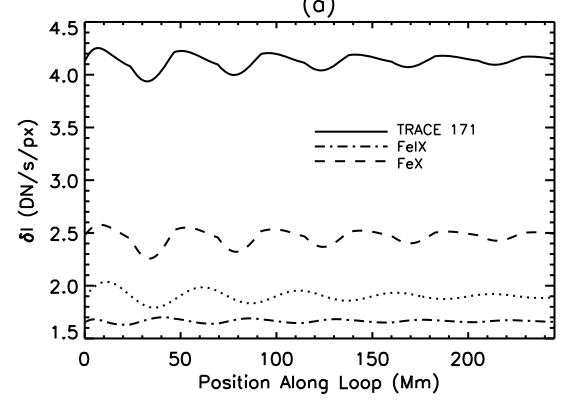

(b)

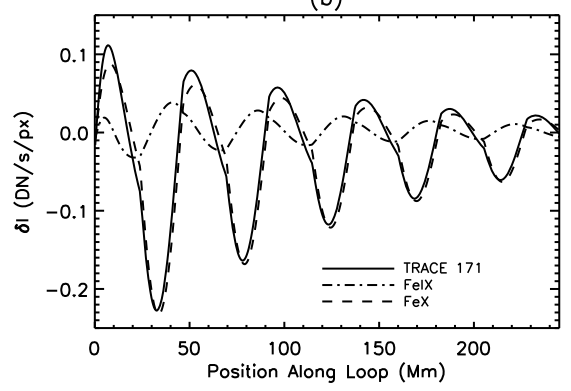

Fig. 8. Synthesised emission of TRACE $171 \AA$ for uniform equilibrium temperature and density. a) TRACE $171 \AA$ intensity (solid) with the intensity due to Fe IX (dot-dashed) and Fe X (dashed). The density from Fig. 3a is renormalised and plotted (dotted) to illustrate the phase difference when compared to the Fe IX intensity. The units of $\delta I$ do not apply to the dotted line. b) Same as a) but with the constant background subtracted to give the perturbed intensity.

stratified medium with an equilibrium temperature gradient (i.e. Fig. 7), we first need to understand the situation of a uniform temperature and density.

\subsection{Uniform equilibrium temperature and density}

The intensity detected from any instrument passband depends on many different factors, as mentioned above and shown by Eq. (44). The total intensity in the passband is the sum of the intensities of all spectral lines that emit in the wavelength range of the instrument response function. The intensity of each line mainly depends on the loop density squared, as well as the ionisation balance and the emissivity of the spectral lines, both of which are temperature dependent. In order to understand how these quantities affect the resulting emission, we use the example of TRACE $171 \AA$. We first look at the individual spectral lines that contribute to the emission. The ionisation balance and the emissivity for each line is then examined. By comparing these quantities and the loop density we gain an understanding of how each factor contributes to the overall emission.

We initially forward model the simulation results shown in Fig. 3a of a slow wave propagating in a unstratified medium at a uniform equilibrium temperature of $1 \mathrm{MK}$ which is damped by thermal conduction. Figure 8 a shows the resulting synthesised emission as would be observed in the TRACE $171 \AA$ passband. As TRACE is an imager, it has a wide spectral coverage, with the instrument response function covering wavelengths from around $160 \AA$ to $190 \AA$, observing radiation emitted from a number of iron and oxygen lines. The solid line in Fig. 8a gives the intensity from all the emission lines in the $171 \AA$ passband, where the main contribution comes from the Fe IX and Fe X ions. The intensities, by considering only Fe IX or Fe X, are shown by the dot-dashed and dashed lines, respectively. For this

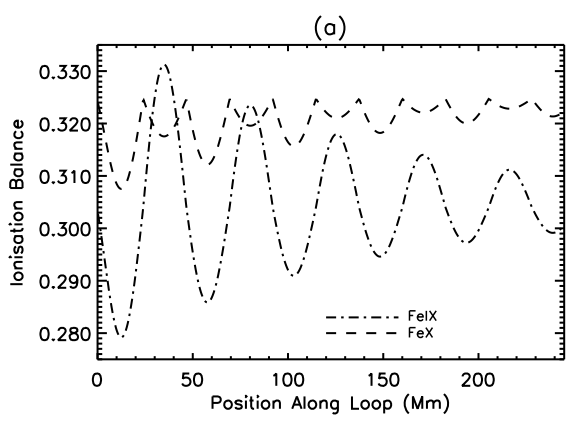

(b)

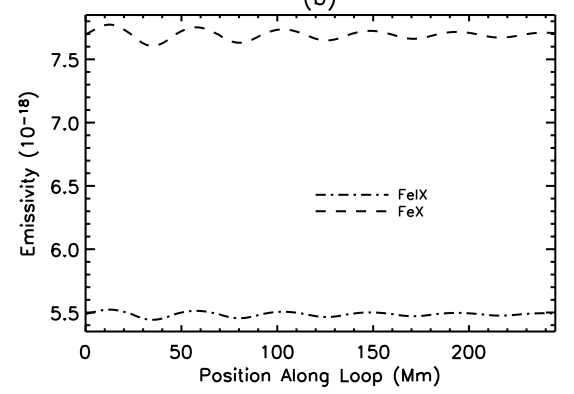

Fig. 9. Contributions of Fe IX and Fe X to TRACE $171 \AA$ A. a) Ionisation balance and b) emissivity for Fe IX (dot-dashed) and Fe X (dashed).

example, at $1 \mathrm{MK}$, the other lines in the $171 \AA$ passband make a negligible contribution to the emission and are not mentioned again. The solid line shows an oscillation around a background of $\sim 4.1 \mathrm{DN} \mathrm{s}^{-1}$ pixel $^{-1}$, the total intensity of this line is the sum of the Fe IX and FeX intensities. Clearly the FeX line makes the largest contribution as it oscillates around a background of $\sim 2.5 \mathrm{DN} \mathrm{s}^{-1}$ pixel $^{-1}$, compared to only $\sim 1.6 \mathrm{DN} \mathrm{s}^{-1}$ pixel $^{-1}$ for Fe IX. From Fig. 8a it is difficult to determine the phase, amplitude and shape of the oscillations in each of the three cases. To bring out these quantities, the constant background of each line has been subtracted, i.e. $\delta I=I-I(x=0)$ and is plotted in Fig. 8b.

From Fig. 8b, it is again evident that Fe $\mathrm{X}$ (dashed) is the dominant line, as the total oscillation (solid) has a very similar shape, amplitude and phase. However, as shown by Fig. 8a, Fe IX (dot-dashed) still contributes and has a very different shape of oscillation, which is in fact out of phase with the other intensities and the simulation density perturbation. Both Fe IX and $\mathrm{Fe} X$ variations result from the same oscillation, i.e. they have the same input energy and density. Therefore, any observed phase difference, etc, is a consequence of the atomic physics producing the observed radiation rather than the physical conditions (i.e. temperature and density) of the loop. These effects are mainly due to the ionisation balance and the emissivity and must be understood in order to correctly interpret any observed intensity oscillations. The ionisation balance is a measure of the amount of each ion in a particular ionisation state. For example, at a temperature of $\approx 6 \times 10^{5} \mathrm{~K}, \mathrm{Fe}$ is mainly in the form of Fe IX. However, at a temperature of $\approx 1 \times 10^{6} \mathrm{~K}$ there is more Fe $\mathrm{X}$. The emissivity is a measure of how much radiation is emitted by these ion species at a particular temperature, density and wavelength.

To understand the shape of the resulting oscillations in Fe IX and Fe X shown by Fig. 8b we examine the ionisation balance and the emissivity individually for both Fe IX and Fe X. The ionisation balance for Fe IX (dot-dashed) and Fe X (dashed) is shown in Fig. 9a, with Fig. 9b showing the emissivity. The emissivity for both Fe IX and Fe X shows oscillations in phase with the simulation results shown by the dot-dashed line in Fig. 3a. This is 


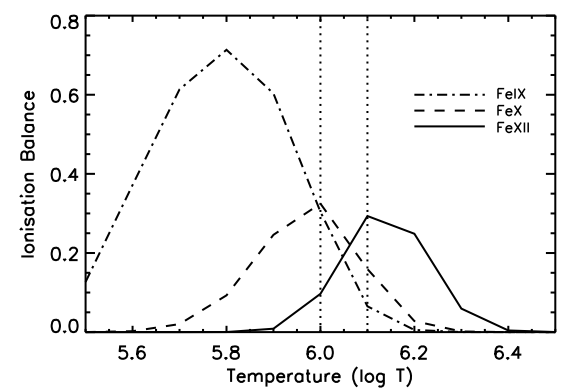

Fig. 10. Ionisation balance as a function of temperature. Fe IX (dotdashed), FeX (dashed) and Fe XII (solid). Vertical dotted lines represent $T_{0}$ of $1 \mathrm{MK}$ and $1.25 \mathrm{MK}$.

a result of the density perturbation, which when positive means there are more ions to emit radiation and hence the emissivity is increased. The converse is true when the density perturbation is negative. For this example the emissivity is found to have little effect on the shape of the oscillation, merely the magnitude of the total emission, $I$. It is the combination of the loop density squared and the ionisation balance which gives the oscillation its shape and phase. The ionisation balance depends on the temperature perturbation, as this changes the amount of each element that is in a particular ionisation state. Again, the more ions in a particular state, the more radiation is emitted, hence the stronger the intensity. Both dashed and dot-dashed lines in Fig. 9a are out of phase with the simulation results, shown as the energy perturbation by the dashed line in Fig. 3a. Fe IX is $180^{\circ}$ out of phase with the energy/temperature perturbation, whereas $\mathrm{Fe} \mathrm{X}$ is more complicated with a very different shape. This behaviour is consistent with that found by De Moortel \& Bradshaw (2008) who investigated the effects of the ionisation balance and the emissivity, for uniform equilibrium temperature and density, on intensity perturbations. They also include the effects of non-linearity.

As discussed earlier, the oscillation shapes in Fig. 8b are, in this case, combinations of the density and the ionisation balance. The population fraction of Fe IX is $180^{\circ}$ out of phase with the density which, as shown by Fig. $8 \mathrm{~b}$, produces emission with a phase between the two. The emission of Fe X, however, is in phase with the density but shows some asymmetry towards negative perturbation amplitudes (negative after the background is subtracted). This suggests that it is the density that dominates the emission with the ionisation causing a slight asymmetry.

The shape of the ionisation balances in Fig. 9a can be explained by looking at the formation temperatures of Fe IX and FeX. These are shown by the dot-dashed and dashed lines in Fig. 10 for Fe IX and Fe X respectively. The intersection of the vertical dotted line at $\log T=6$ with the dot-dashed and dashed lines gives the ionisation balance for $\mathrm{Fe} I X$ and $\mathrm{FeX}$, respectively at $1 \mathrm{MK}$ (this is the equilibrium value from the simulations about which the temperature oscillates). From Fig. 10 it is clear that the ionisation balances for both Fe IX and Fe X are comparable at this temperature, hence they both contribute to the full TRACE $171 \AA$ intensity. However, as shown by Fig. 9b, the emissivity of Fe X is higher than Fe IX and, therefore, Fe X has the stronger contribution to the magnitude of the emission, as shown in Fig. 8a. The ionisation balances in Fig. 9a depend on the equilibrium temperature of the oscillations, for example, at $1 \mathrm{MK}$ the ionisation balance of Fe IX, in Fig. 10, has a negative gradient, whereas it is at the peak of FeX. Considering Fe IX, if the temperature increases the ionisation balance decreases (Fig. 10), whereas, a decrease in temperature produces more Fe IX. This explains why the dot-dashed line in Fig. 9a has

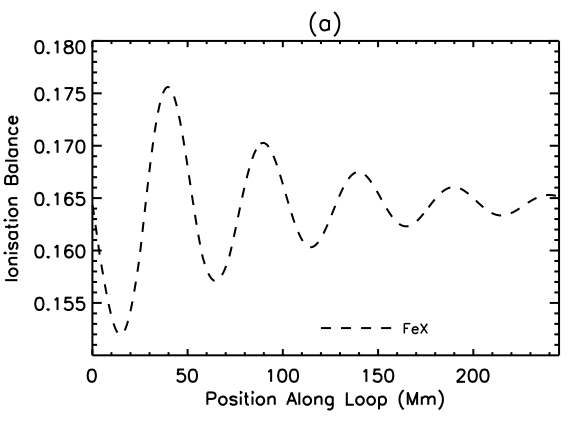

(b)

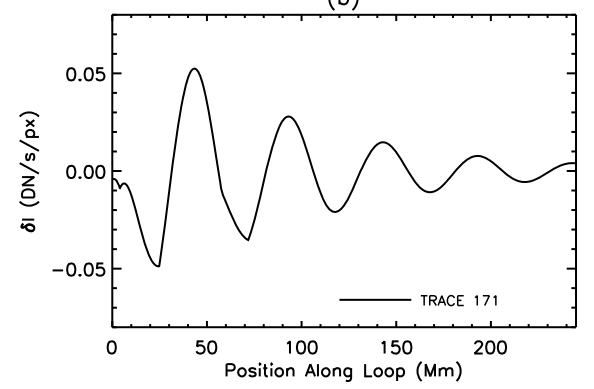

Fig. 11. Synthesised emission for a uniform temperature of 1.25 MK. a) Ionisation balance of $\mathrm{Fe} \mathrm{X}$ and b) total TRACE $171 \AA$ emission.

the opposite phase to the simulation results. Fe $\mathrm{X}$ is at the peak at $1 \mathrm{MK}$ and therefore, any oscillation produces a decrease in Fe X. However, the gradient of the ionisation balance for temperatures above $1 \mathrm{MK}$ is steeper than that below $1 \mathrm{MK}$. As a result, a negative perturbation in temperature reduces the ionisation balance less than a positive perturbation of the same amplitude.

The loop density in addition to the ionisation balance and the emissivity of both Fe IX and Fe X contributes to the magnitude of the emission. For this particular example it is the effects of the density on the Fe X emission that mostly contributes to the phase of the oscillation in the synthesised emission in Fig. 8a. The emissivity and the ionisation balance have little effect on this phase, however, the ionisation balance does affect the oscillation shape by causing an asymmetry.

By looking at the same oscillation, but about an equilibrium temperature of $1.25 \mathrm{MK}$, produces some what different results. The ionisation balance for Fe $\mathrm{X}$ oscillating about $1.25 \mathrm{MK}$ is shown in Fig. 11a and is $180^{\circ}$ out of phase with the temperature and density perturbations. This is due to the negative gradient of the Fe X ionisation balance at 1.25 MK, as shown in Fig. 10. For this case, when considering the shape and phase of the oscillation, as shown in Fig. $11 \mathrm{~b}$, it is the ionisation balance that dominates the density and the emissivity. This results in emission for TRACE $171 \AA$ which is out of phase with the simulation oscillations. Due to the increase in temperature compared to the previous example, Fe IX has a much weaker contribution to the full $171 \AA$ emission due to a reduced ionisation balance, as seen in Fig. 10. Such a case demonstrates the difficulties in correctly interpreting observational data, where for this example the observed intensity variation would appear out of phase with the density and temperature/energy perturbations. This is discussed in more detail in De Moortel \& Bradshaw (2008).

Figure $8 \mathrm{~b}$ (solid) shows an oscillation that is slowly damped (due to thermal conduction) along a coronal loop, but is still clearly visible at $200 \mathrm{Mm}$. In contrast to this, observed oscillations are only ever detected to around $10 \mathrm{Mm}$ (De Moortel et al. 2002c). We now examine the observation consequences of including gravitational stratification. 
(a)

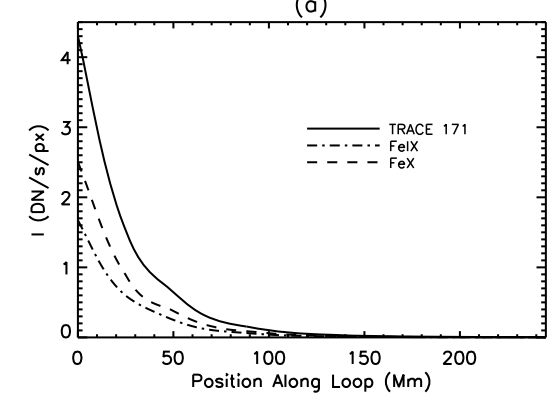

(b)

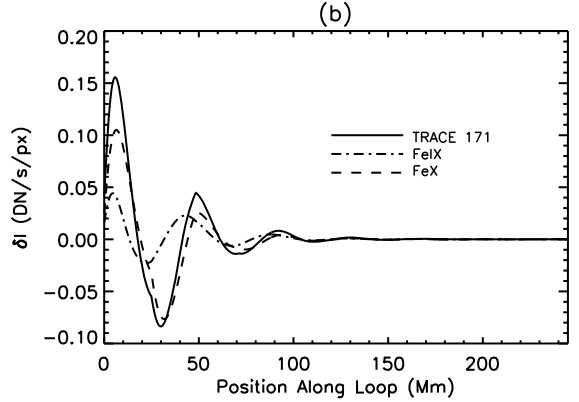

Fig. 12. Synthesised emission of TRACE $171 \AA$ for uniform equilibrium temperature in a gravitationally stratified medium. a) TRACE $171 \AA$ intensity (solid) with the intensity due to Fe IX (dot-dashed) and $\mathrm{Fe} \mathrm{X}$ (dashed). b) same as a) but with the exponentially decreasing background subtracted to give the perturbed intensity.

\subsection{Uniform equilibrium temperature and gravity}

We now forward model the simulation results shown in Fig. 6b of a slow wave propagating in a gravitationally stratified medium at a uniform equilibrium temperature of $1 \mathrm{MK}$ which is damped by thermal conduction. The solid line in Fig. 12a shows the resulting emission for the entire TRACE $171 \AA$ A passband. The synthesised emission by considering only Fe IX or Fe X is shown by the dot-dashed and dashed lines, respectively.

As discussed in Sect. 4.2, the observed intensity depends on the loop density squared. Considering that the inclusion of gravity produces an exponential decrease in the background density (discussed in Sect. 3.1.1), the resulting intensity also decreases exponentially. This can be clearly seen in Fig. 12a. In order to detect oscillations we subtract the background intensity as in Sect. 4.2 for Fig. 8b. However, this is no longer a constant value due to the inclusion of gravity. Consequently, the equilibrium conditions i.e. $\rho_{0}=\rho(t=0, x)$ and $\epsilon_{0}=\epsilon(t=0, x)$ are forward modelled and the subsequent emission is subtracted from Fig. 12a. The results are plotted in Fig. 12b where the solid line represents TRACE $171 \AA$ and the dot-dashed and dashed lines give the Fe IX and Fe $\mathrm{X}$ emission, respectively.

The density and temperature at the loop footpoints of Figs. $8 b$ and $12 b$ are the same. The difference is that for Fig. $12 b$ the density decreases exponentially along the loop. Therefore, as expected the initial peak in both figures is very similar as both results have the same combination of loop density, ionisation balance and emissivity. However, the oscillations in Fig. 12b decay much more rapidly than those in Fig. $8 b$ and do not have the same asymmetry. The oscillations shown in Fig. 12b are clearly only detected within the first $50 \mathrm{Mm}$ to $100 \mathrm{Mm}$. This is an upper limit considering that the effect of noise has been neglected. This is in contrast to Fig. 8b, where an oscillation is visible beyond $200 \mathrm{Mm}$. Both simulation results, shown in Fig. 6b and Fig. 3a, with and without gravity, respectively, show oscillations

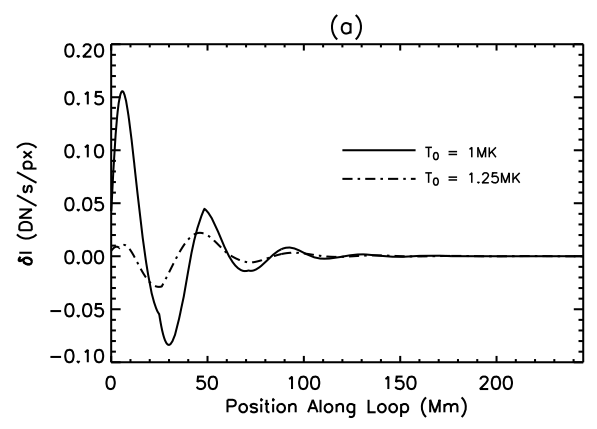

(b)

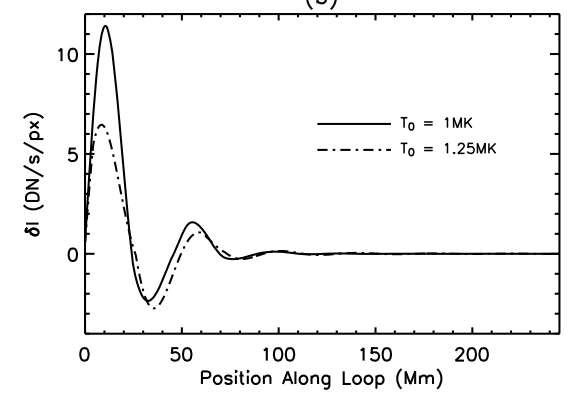

Fig. 13. Synthesised emission for uniform equilibrium temperatures of $1 \mathrm{MK}$ and 1.25 MK. a) TRACE $171 \AA$ and b) EIS Fe XII (195 $)$ ).

of similar amplitude beyond $200 \mathrm{Mm}$. Therefore, the decay of the oscillation in Fig. 12b must be an observational effect due to the exponential decay in density, where the emission drops below the sensitivity of TRACE $171 \AA$. The asymmetry is reduced because the decrease in density is dominant over the ionisation balance.

The simulation results shown in Fig. $6 \mathrm{~b}$ with thermal conduction and gravity for a uniform equilibrium temperature of $1 \mathrm{MK}$ were repeated for a temperature of $1.25 \mathrm{MK}$ with a corresponding density of $1.2 \times 10^{-12} \mathrm{~kg} \mathrm{~m}^{-3}$. This represents the maximum equilibrium temperature of the profiles shown in Fig. 1. The simulation results are forward modelled for TRACE $171 \AA$ and are shown by the dot-dashed line in Fig. 13a. The solid line in Fig. 13a is the same as in Fig. 12b and gives the emission for a uniform equilibrium temperature of $1 \mathrm{MK}$. As discussed in Sect. 4.2, the emission for TRACE $171 \AA$ at $1.25 \mathrm{MK}$ (for this example) is out of phase with the $1 \mathrm{MK}$ emission. This can be clearly seen by comparing the solid and dot-dashed lines in Fig. 13a. The 1.25 MK emission has a much lower magnitude than that of the $1 \mathrm{MK}$ due to two effects. Firstly, the density is lower (to keep constant pressure) which reduces the emission, as discussed earlier. Secondly, the ionisation balance of Fe IX and $\mathrm{Fe} \mathrm{X}$ at this temperature is reduced, as shown in Fig. 10 and discussed in Sect. 4.2. The reduction in magnitude of the dotdashed line in Fig. 13a compared to the solid line in Fig. 11b is due purely to the difference in background density.

To complement the imager TRACE we examine the emission from the spectrometer EIS on Hinode. Figure 13b gives the emission of both $1 \mathrm{MK}$ and 1.25 MK for the Fe XII (195 $\AA$ ) line by the solid and dot-dashed lines, respectively. The background emission is subtracted as described for Fig. 12b. The relative scales show the higher sensitivity of EIS compared to TRACE $171 \AA$. Fe XII has a peak formation temperature of around 1.25 MK as shown by the solid line in Fig. 10, despite this, in Fig. 13b the $1 \mathrm{MK}$ emission has a greater magnitude than that of $1.25 \mathrm{MK}$. This difference is a result of the reduced density of the $1.25 \mathrm{MK}$ case. Comparison between TRACE $171 \AA$ 
and EIS Fe XII for the 1 MK case show very similar oscillations, however, for 1.25 MK the resulting oscillations appear substantially different. This highlights the importance of observing a range of emission lines for different instruments in order to ease the interpretation of observational data. The results for a uniform temperature equilibrium of thermal conduction in a stratified medium, as shown in Fig. 13, for TRACE $171 \AA$ and Hinode/EIS Fe XII ( $195 \AA$ ) are consistent with a rapidly decaying oscillation visible to the order of $50 \mathrm{Mm}$, as shown by observations, e.g. De Moortel et al. (2002c,a).

Each emission line is sensitive to a certain temperature range, outside of which no emission will be observed. By using a uniform temperature equilibrium with moderate temperature perturbations $(\sim 5 \%)$ the entire loop is at approximately the same temperature. Consequently, for a uniform temperature equilibrium, either the whole loop is observed by an instrument/emission line or none of the loop is. This is in contrast to the results for a non-uniform temperature equilibrium, which are discussed in Sect. 4.4.

\subsection{Non-uniform equilibrium temperature}

By including a non-uniform equilibrium temperature profile observations with a particular instrument or emission line will only observe part of the loop. Imagers such as TRACE observe over broad temperature ranges (with a range of emission lines) and hence are expected to detect emission along a greater length of the loop than that of a spectrometer using a single emission line.

In this section the simulation results shown in Fig. 7b using the temperature profile given by the solid line in Fig. 1b are forward modelled for a range of instruments and emission lines: TRACE $171 \AA$ is sensitive to transition region and coronal temperatures and should detect emission along most of the loop length; EIS Mg VI(269 $\AA$ ) is sensitive to temperatures of around $0.4 \mathrm{MK}$; and Hinode/EIS Fe XII(195 $\AA$ ) and SoHO/CDS Mg X (624 $\AA)$ are sensitive to coronal temperatures around 1.25 MK. The spectrometers are sensitive to single emission lines (for these examples) and therefore observe more localised emission.

The background emission has been subtracted from each figure in this section, as described in Sect. 4.3. Figure 14a shows the synthesised emission for TRACE $171 \AA$ A with Fig. 14b showing the same, only plotted for a different scale of $\delta I$. It is clear from Fig. 14b that as expected the TRACE $171 \AA$ detects oscillations in the entire loop. However, it is the transition region which dominates, with the observed signal around two orders of magnitude greater than the coronal region.

There is some observational evidence for larger amplitudes in the transition region. Marsh et al. (2003) detected propagating oscillations with a five minute periodicity with amplitudes of $9.8 \pm 3.1 \%$ and $8.6 \pm 1.2 \%$ in the chromosphere and the corona, respectively, compared to $12.4 \pm 2.1 \%$ in the transition region. Brynildsen et al. (2003) detected three minute oscillations and found amplitudes of $7-16 \%$ in the transition region, compared to only $2.5-6 \%$ and $3-5 \%$ in the chromosphere and corona, respectively. O'Shea et al. (2002) also detected three minute oscillations and found the amplitude to peak in the transition region. These observations are consistent with the model in that they do show higher oscillation amplitudes in the transition region than the corona, however, the difference in intensity is much less than predicted by our model. The reason for this discrepancy remains unclear. (a)

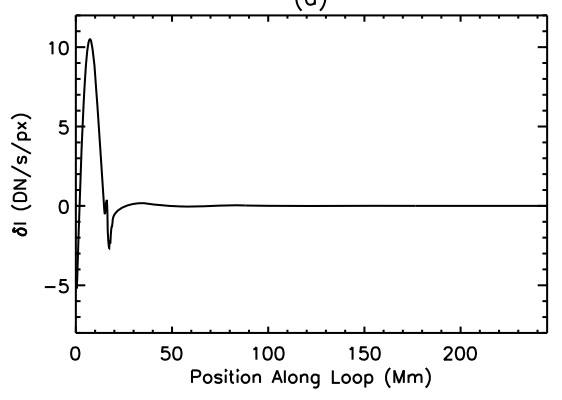

(b)

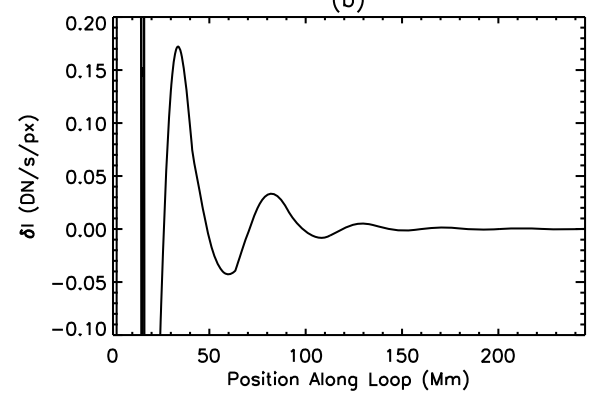

Fig. 14. Synthesised emission for non-uniform equilibrium temperature. a) TRACE $171 \AA$, b) same as a) but plotted for $-0.1 \leq \delta I \leq 0.2$ to highlight the coronal oscillations.

The results of the spectrometers are shown in Fig. 15a-c for EIS Mg VI, EIS Fe XII and CDS Mg X, respectively. The relative scales of Fig. 15a and b compared the Fig. 15c show the higher sensitivity of EIS compared to CDS. As expected the spectrometers observe more localised regions of the loop compared to that of TRACE shown in Fig. 14. The Mg VI line shows a spike in the transition region but no oscillation in the corona. Neither the Fe XII or Mg X lines observe emission from the low transition region. However, both show spikes in the upper transition region/low corona followed by a rapidly decaying oscillation in the corona. This is consistent with TRACE $171 \AA$ from Fig. 14. The oscillations in TRACE and EIS Fe XII are $180^{\circ}$ out of phase with each other, with the CDS Mg X line showing a phase between the two. This demonstrates the difficulty in correctly interpreting the density and temperature perturbations along the loop from observations.

\section{Discussion and conclusions}

We have numerically simulated the propagation of slow waves, of periodicity five minutes, in $1 \mathrm{D}$, through the transition region and the corona. The results of a uniform temperature equilibrium are consistent with previous work by De Moortel \& Hood (2003, 2004). The effects of thermal conduction on the propagation of slow waves is investigated. The damping caused by this mechanism is again consistent with previous work by De Moortel \& Hood (2003). Beyond this, it was found that the inclusion of thermal conduction produces a phase shift at the lower boundary between the perturbations in velocity, density and energy/temperature. This phase shift remains constant for waves propagating in a medium of uniform equilibrium temperature. However, the propagation speed depends on the equilibrium temperature, hence for a non-uniform equilibrium temperature this phase shift varies along the loop. The phase shift depends on both the energy and the density of the region and may have observational consequences. Intensity observations depend on the energy/temperature in contrast to the velocity for Doppler shift 
(a)

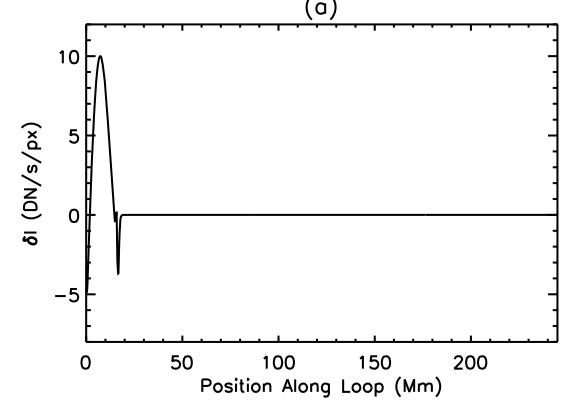

(b)

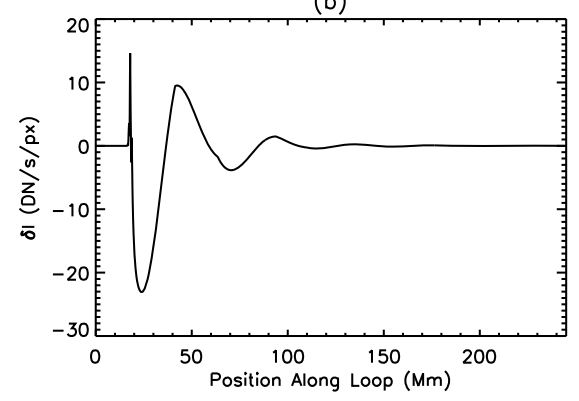

(c)

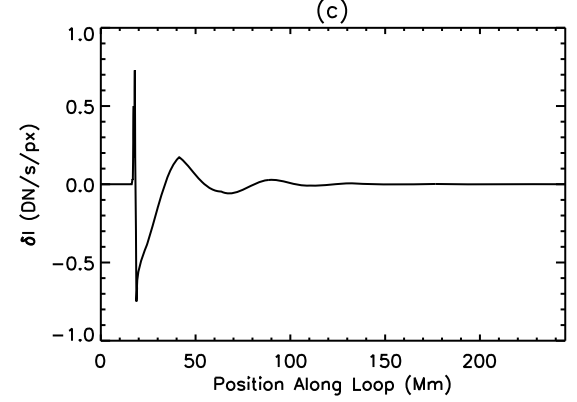

Fig. 15. Synthesised emission for non-uniform equilibrium temperature. a) EIS Mg VI (269 ̊), b) EIS Fe XII (195 ^) and c) CDS $\operatorname{Mg}$ X (624 ̊).

observations. Consequently, the phase shift between these three quantities may be observable as a phase shift between intensity and velocity observations. However, Doppler velocity observations are beyond the scope of the present work.

The effects of non-uniform equilibrium temperature are investigated by the inclusion of a gradient from a transition region temperature of $\sim 0.3 \mathrm{MK}$ to a coronal value of $1.25 \mathrm{MK}$. Thermal conduction and gravitational stratification are included. The wave amplitudes grow in the transition region and are damped in the corona. The steep transition region causes localised spikes in the energy and density perturbations where the temperature and density gradients are largest. The transition region also causes reflection of the wave. The temperature profile used has a transition region with a height of around $5 \mathrm{Mm}$, however, a more realistic value may be $1-2 \mathrm{Mm}$. This would increase the temperature and density gradients and may, therefore, increase the spikes produced.

Using the technique of forward modelling the density and energy from the simulations are used to calculate the synthesised emission observed for TRACE $171 \AA$ and the spectrometer lines EIS Fe XII, EIS Mg VI and CDS Mg X. It is shown for the case of different uniform equilibrium temperatures that despite the same input oscillations the observed phase, amplitude and shape of the wave can be different. These effects are a complicated combination of the loop density, the ionisation balance and the emissivity and depend on the temperature of the loop. It is shown that an oscillation of around 5\% of a background temperature of 1.25 MK observed by TRACE $171 \AA$ and EIS Fe XII would appear $180^{\circ}$ out of phase.

The dependence of intensity on the loop density squared means that in a gravitationally stratified medium the oscillations quickly fall below instrument sensitivity and can only be detected to around $50 \mathrm{Mm}$, compared to over $200 \mathrm{Mm}$ without gravity. This value is of the order of that observed for propagating slow waves (around $10 \mathrm{Mm}$, De Moortel et al. 2002c) but is still high. However, it is an upper limit in the detection length as observations have noise to consider which will reduce the detection threshold.

The inclusion of an inclination angle for magnetic field lines, as shown by De Pontieu et al. (2005), allows leakage of five minute p-modes through the upper photosphere and the chromosphere. We consider only the transition region and the corona and hence leave the inclusion of an inclination angle at the solar surface to future study. Inclined magnetic field lines would reduce the effects of gravitational stratification on increasing the wave amplitude and hence may reduce damping lengths to values closer to observations.

With the addition of an equilibrium temperature gradient the forward modelling results vary more between imagers and spectrometers. TRACE $171 \AA$ A observes oscillations along the entire loop, however, the coronal part is weak with emission from the transition region dominant. Low data counts for coronal oscillations are expected for TRACE, where oscillations have been observed by spatial and temporal averaging (e.g. De Moortel et al. 2002c; McEwan \& De Moortel 2006). However, a feature which has not been observed is the dominant emission from the transition region suggested by Fig. 14. The reason for this discrepancy is unclear and remains an open question.

By using a range of spectral lines, waves can be observed as they propagate through the solar atmosphere. The emission detected by the spectrometers is more localised than TRACE, detecting either the transition region or the corona as expected. No coronal oscillation is visible in the EIS Mg VI line which is sensitive to transition region temperatures. The EIS Fe XII and CDS Mg X lines have similar peak formation temperatures of around 1.25 MK and as a result detect similar coronal oscillations. They are also consistent with the coronal part of TRACE $171 \AA$ An In addition, both the spectrometer emission lines observe a spike in the high transition/low corona. TRACE, EIS Fe XII and CDS Mg X all show rapidly decaying oscillations observable to around $50 \mathrm{Mm}$. However, the phase of the oscillation is different for each line, with TRACE and Fe XII completely out of phase. This demonstrates the difficulty in interpreting the density and temperature perturbations from observed oscillations. The comparison of many different lines from different instruments is important to correctly interpret observations of such oscillations, as are simultaneous Doppler velocity observations.

Acknowledgements. N.R.O and I.D.M acknowledge support of an STFC Ph.D. studentship and a Royal Society University Research Fellowship, respectively. The authors would also like to thank both the referee and Dr. D. Bewsher for their useful comments in improving the manuscript.

\section{References}

Arber, T. D., Longbottom, A. W., Gerrard, C. L., \& Milne, A. M. 2001, J. Comp. Phys., 171, 151

Aschwanden, M. J. 2004, in SOHO 15 Coronal Heating, ed. R. W. Walsh, J. Ireland, D. Danesy, \& B. Fleck, ESA SP, 575, 97 
Berghmans, D., \& Clette, F. 1999, Sol. Phys., 186, 207

Bradshaw, S. J., \& Mason, H. E. 2003, A\&A, 407, 1127

Brynildsen, N., Maltby, P., Kjeldseth-Moe, O., \& Wilhelm, K. 2003, A\&A, 398, L15

Culhane, J. L., Harra, L. K., James, A. M., et al. 2007, Sol. Phys., 243, 19

De Moortel, I. 2005, Roy. Soc. London Philos. Trans. Ser. A, 363, 2743

De Moortel, I. 2006, Roy. Soc. London Philos. Trans. Ser. A, 364, 461

De Moortel, I., \& Hood, A. W. 2003, A\&A, 408, 755

De Moortel, I., \& Hood, A. W. 2004, A\&A, 415, 705

De Moortel, I., \& Bradshaw, S. J. 2008, Sol. Phys., 139

De Moortel, I., Ireland, J., \& Walsh, R. W. 2000, A\&A, 355, L23

De Moortel, I., Hood, A. W., Ireland, J., \& Walsh, R. W. 2002a, Sol. Phys., 209, 89

De Moortel, I., Ireland, J., Hood, A. W., \& Walsh, R. W. 2002b, A\&A, 387, L13

De Moortel, I., Ireland, J., Walsh, R. W., \& Hood, A. W. 2002c, Sol. Phys., 209, 61

De Moortel, I., Hood, A. W., Gerrard, C. L., \& Brooks, S. J. 2004, A\&A, 425, 741

De Pontieu, B., Erdélyi, R., \& De Moortel, I. 2005, ApJ, 624, L61

Deforest, C. E., \& Gurman, J. B. 1998, ApJ, 501, L217

Delaboudinière, J.-P., Artzner, G. E., Brunaud, J., et al. 1995, Sol. Phys., 162, 291

Dere, K. P., Landi, E., Mason, H. E., Monsignori Fossi, B. C., \& Young, P. R. 1997, A\&AS, 125, 149

Domingo, V., Fleck, B., \& Poland, A. I. 1995, Sol. Phys., 162, 1

Erdélyi, R., Petrovay, K., Roberts, B., \& Aschwanden, M. 2003, Turbulence, Waves and Instabilities in the Solar Plasma, ed. R. Erdélyi, K. Petrovay, B. Roberts, \& M. Aschwanden (Kluwer Academic Publishers)

Feldman, U., Mandelbaum, P., Seely, J. F., Doschek, G. A., \& Gursky, H. 1992, ApJS, 81, 387

Handy, B. N., Acton, L. W., Kankelborg, C. C., et al. 1999, Sol. Phys., 187, 229

King, D. B., Nakariakov, V. M., Deluca, E. E., Golub, L., \& McClements, K. G. 2003, A\&A, 404, L1

Klimchuk, J. A., Tanner, S. E. M., \& De Moortel, I. 2004, ApJ, 616, 1232

Kohl, J. L., Esser, R., Gardner, L. D., et al. 1995, Sol. Phys., 162, 313

Luciani, J. F., Mora, P., \& Virmont, J. 1983, Phys. Rev. Lett., 51, 1664

Marsh, M. S., \& Walsh, R. W. 2006, ApJ, 643, 540
Marsh, M. S., Walsh, R. W., De Moortel, I., \& Ireland, J. 2003, A\&A, 404, L37 Mazzotta, P., Mazzitelli, G., Colafrancesco, S., \& Vittorio, N. 1998, A\&AS, 133, 403

McEwan, M. P., \& De Moortel, I. 2006, A\&A, 448, 763

Nakariakov, V. M., \& Verwichte, E. 2005, Living Rev. Sol. Phys., 2, 3

Nakariakov, V. M., Verwichte, E., Berghmans, D., \& Robbrecht, E. 2000, A\&A, 362,1151

Nightingale, R. W., Aschwanden, M. J., \& Hurlburt, N. E. 1999, Sol. Phys., 190, 249

Ofman, L., Romoli, M., Poletto, G., Noci, G., \& Kohl, J. L. 1997, ApJ, 491, L111

Ofman, L., Nakariakov, V. M., \& Deforest, C. E. 1999, ApJ, 514, 441

Ofman, L., Romoli, M., Poletto, G., Noci, G., \& Kohl, J. L. 2000, ApJ, 529, 592

O'Shea, E., Muglach, K., \& Fleck, B. 2002, A\&A, 387, 642

Peter, H., Gudiksen, B. V., \& Nordlund, Å. 2006, ApJ, 638, 1086

Priest, E. R. 1982, Solar magneto-hydrodynamics (Dordrecht, Holland; Boston:

D. Reidel Pub. Co., Hingham), 74P

Robbrecht, E., Verwichte, E., Berghmans, D., et al. 2001, A\&A, 370, 591

Roberts, B. 1985, Magnetohydrodynamic Waves, Chapter 3 in Solar System Magnetic Fields, 37 (D Reidel)

Roberts, B. 2004, in SOHO 13 Waves, Oscillations and Small-Scale Transients Events in the Solar Atmosphere: Joint View from SOHO and TRACE, ed. H. Lacoste, ESA SP, 547, 1

Roberts, B., Edwin, P. M., \& Benz, A. O. 1984, ApJ, 279, 857

Schrijver, C. J., Title, A. M., Berger, T. E., et al. 1999, Sol. Phys., 187, 261

Shelyag, S., Erdélyi, R., \& Thompson, M. J. 2006, ApJ, 651, 576

Taroyan, Y., \& Bradshaw, S. 2008, A\&A, 481, 247

Taroyan, Y., Erdélyi, R., Wang, T. J., \& Bradshaw, S. J. 2007, ApJ, 659, L173

Tsiklauri, D., \& Nakariakov, V. M. 2001, A\&A, 379, 1106

Uchida, Y. 1970, PASJ, 22, 341

Wang, T. J. 2004, in SOHO 13 Waves, Oscillations and Small-Scale Transients Events in the Solar Atmosphere: Joint View from SOHO and TRACE, ed. H. Lacoste, ESA SP, 547, 417

Young, P. R., Landi, E., Bromage, G. E., et al. 2003, in The Future of Cool-Star Astrophysics: 12th Cambridge Workshop on Cool Stars, Stellar Systems, and the Sun (2001 July 30-August 3), ed. A. Brown, G. M. Harper, \& T. R. Ayres, University of Colorado, 1119 